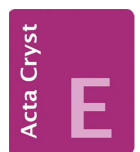

CRYSTALLOGRAPHIC COMMUNICATIONS

ISSN 2056-9890

Received 18 July 2016

Accepted 31 August 2016

Edited by W. T. A. Harrison, University of Aberdeen, Scotland

Keywords: crystal structure; 4-hydroxybenzoate; 4-oxidobenzoate; 1,10-phenanthroline; copper; dinuclear complex cation.

CCDC reference: 1501982

Supporting information: this article has supporting information at journals.iucr.org/e

\section{Crystal structure of $\mu$-4-oxidobenzoato- $\kappa^{2} O^{1}: O^{4}$ - bis[bis $\left(1,10-\right.$ phenanthroline- $\left.\kappa^{2} N, N^{\prime}\right) \operatorname{copper}($ II) $]$ bis(4-hydroxybenzoate) 7.5 -hydrate}

\author{
Jian-Rong Su* and Yu Li
}

School of Pharmaceutical and Chemical Engineering, Taizhou University, People's Republic of China. * Correspondence
e-mail: sujr_tzu@foxmail.com

The title hydrated complex, $\left[\mathrm{Cu}_{2}\left(\mathrm{C}_{7} \mathrm{H}_{4} \mathrm{O}_{3}\right)\left(\mathrm{C}_{12} \mathrm{H}_{8} \mathrm{~N}_{2}\right)_{4}\right]\left(\mathrm{C}_{7} \mathrm{H}_{5} \mathrm{O}_{3}\right)_{2} \cdot 7.5 \mathrm{H}_{2} \mathrm{O}$, is composed of dinuclear $\mathrm{Cu}^{\mathrm{II}}$ complex cations, noncoordinating 4-hydroxybenzoate anions and water molecules of crystallization. In the dinuclear complex cation, the $\mathrm{Cu}^{\mathrm{II}}$ ions are bridged by a 4-oxidobenzoate ligand and thus each metal ion is five-coordinated by two chelating 1,10-phenanthroline (phen) molecules and one anionic $\mathrm{O}$ atom in a distorted trigonal-bipyramid geometry. In the crystal, aromatic $\pi-\pi$ stacking occurs between phen rings of neighbouring dinuclear $\mathrm{Cu}^{\mathrm{II}}$ complex cations, forming two-dimensional supramolecular systems parallel to (100).

\section{Chemical context}

In some biological systems, $\pi-\pi$ stacking between aromatic rings is correlated with the elctron-transfer process (Deisenhofer \& Michel, 1989). To study the effect of $\pi-\pi$ stacking, the title complex, (I), incorporating 1,10-phenanthroline (phen), has been prepared and its crystal structure is presented here.

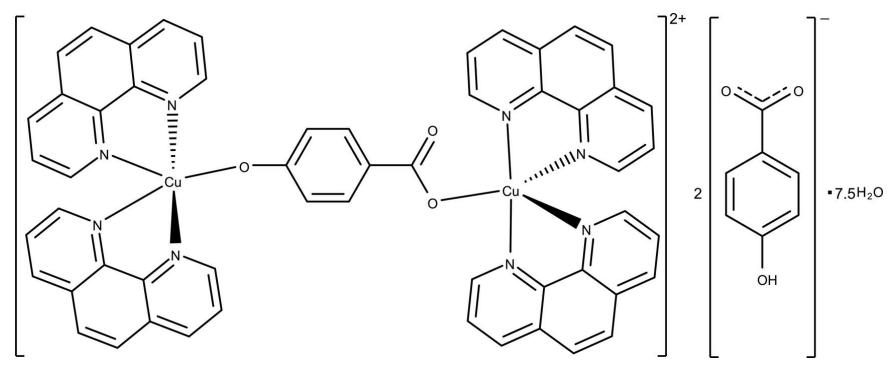

\section{Structural commentary}

The crystal structure of (I) is composed of dinuclear $\mathrm{Cu}^{\mathrm{II}}$ complex cations, noncoordinating 4-hydroxybenzoate anions and solvent water molecules, as shown in Fig. 1. The molecular structure of the dinuclear $\mathrm{Cu}^{\mathrm{II}}$ complex cation is shown in Fig. 2. Two $\mathrm{Cu}^{\mathrm{II}}$ atoms $(\mathrm{Cu} 1$ and $\mathrm{Cu} 2)$ are bridged by one 4oxidobenzoate anion through oxido and carboxy $\mathrm{O}$ atoms (O53 and $\mathrm{O} 51$, respectively), with a $\mathrm{Cu} 1-\mathrm{O} 53$ bond length of 1.941 (3) $\AA$ and a Cu2-O51 bond length of 1.979 (3) $\AA$. Each $\mathrm{Cu}^{\text {II }}$ atom is five-coordinated, displaying a distorted trigonalbipyramidal geometry (Table 1). The $\mathrm{Cu} 1$ atom is coordinated by two chelating phen rings ( $\mathrm{N} 1, \mathrm{~N} 2, \mathrm{~N} 3$ and $\mathrm{N} 4)$ intersecting at an angle of $71.35(5)^{\circ}$. The out-of-plane $\mathrm{Cu} 1-\mathrm{N} 1$ and $\mathrm{Cu}-$ N3 bond lengths are 2.002 (3) and 2.027 (3) Å, respectively, 


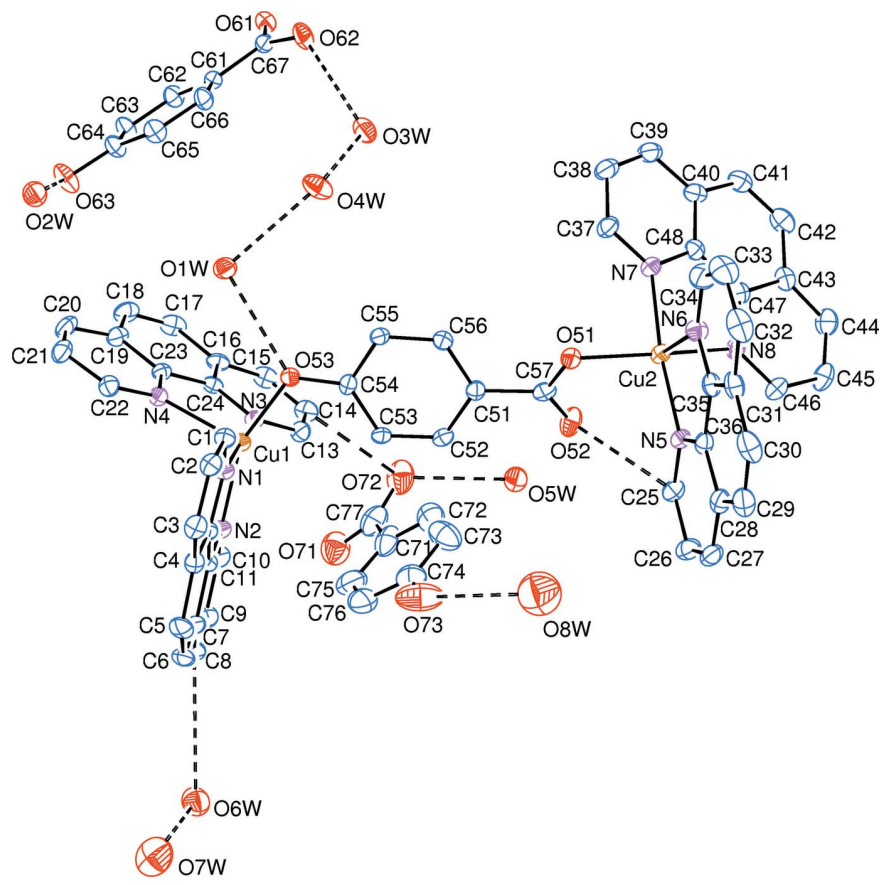

Figure 1

The structures of the molecular entities of (I), shown with $30 \%$ probability displacement ellipsoids. Dashed lines indicate hydrogen bonds. $\mathrm{H}$ atoms have been omitted for clarity.

which are shorter than the in-plane $\mathrm{Cu} 1-\mathrm{N} 2$ and $\mathrm{Cu} 1-\mathrm{N} 4$ bond lengths [2.051 (4) and 2.158 (4) $\AA$, respectively]. The $\mathrm{N} 1-\mathrm{Cu} 1-\mathrm{N} 3$ bond angle is $176.67(15)^{\circ}$. The coordination parameters of the $\mathrm{Cu} 2$ atom are similar to those of the $\mathrm{Cu} 1$ atom. The $\mathrm{Cu}^{\mathrm{II}}$ atoms display apparently different coordination patterns from the $\mathrm{Cu}^{\mathrm{II}}$ complex coordinated by phen and 4-hydroxybenzoate ligands ( $\mathrm{Su}$ et al., 2005), in which the reported complex is mononuclear, with the $\mathrm{Cu}^{\mathrm{II}}$ ion being sixcoordinated by one chelating phen ligand and two chelating 4hydroxybenzoate anions through the carboxylate $\mathrm{O}$ atoms, resulting in an elongated octahedral geometry.

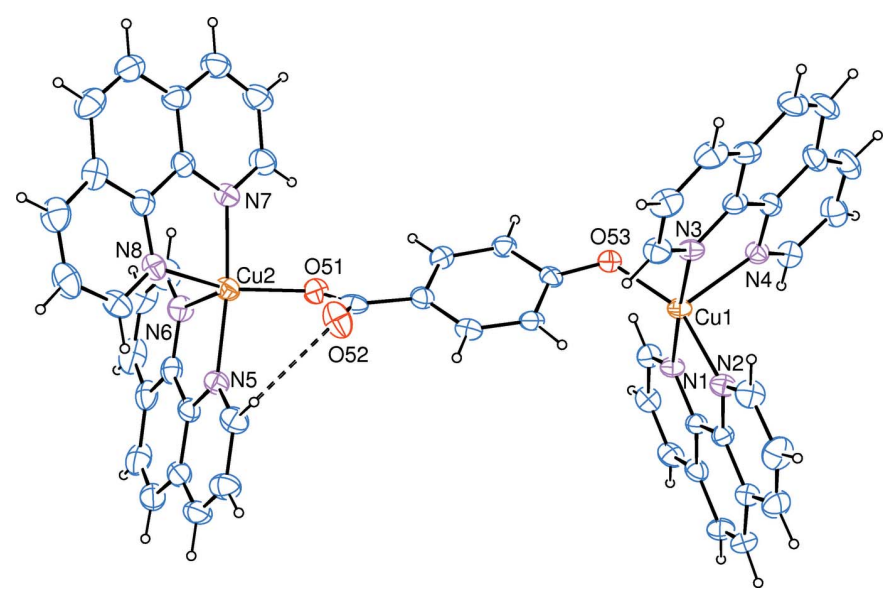

Figure 2

The molecular structure of the dinuclear $\mathrm{Cu}^{\mathrm{II}}$ complex cation in (I), shown with $30 \%$ probability displacement ellipsoids. $\mathrm{H}$ atoms have been omitted for clarity. Dashed lines indicate hydrogen bonds.
Table 1

Selected geometric parameters $\left(\AA,^{\circ}\right)$.

\begin{tabular}{lrlr}
\hline $\mathrm{Cu} 1-\mathrm{O} 53$ & $1.941(3)$ & $\mathrm{Cu} 2-\mathrm{O} 51$ & $1.979(3)$ \\
$\mathrm{Cu} 1-\mathrm{N} 1$ & $2.002(3)$ & $\mathrm{Cu} 2-\mathrm{N} 5$ & $2.010(4)$ \\
$\mathrm{Cu} 1-\mathrm{N} 2$ & $2.051(4)$ & $\mathrm{Cu} 2-\mathrm{N} 6$ & $2.197(4)$ \\
$\mathrm{Cu} 1-\mathrm{N} 3$ & $2.027(3)$ & $\mathrm{Cu} 2-\mathrm{N} 7$ & $2.013(4)$ \\
$\mathrm{Cu} 1-\mathrm{N} 4$ & $2.158(4)$ & $\mathrm{Cu} 2-\mathrm{N} 8$ & $2.079(4)$ \\
& & & \\
$\mathrm{N} 1-\mathrm{Cu} 1-\mathrm{N} 3$ & $176.67(15)$ & $\mathrm{N} 5-\mathrm{Cu} 2-\mathrm{N} 7$ & $173.07(16)$ \\
$\mathrm{O} 53-\mathrm{Cu} 1-\mathrm{N} 2$ & $155.19(14)$ & $\mathrm{O} 51-\mathrm{Cu} 2-\mathrm{N} 5$ & $96.10(15)$ \\
$\mathrm{O} 53-\mathrm{Cu} 1-\mathrm{N} 4$ & $101.39(14)$ & $\mathrm{O} 51-\mathrm{Cu} 2-\mathrm{N} 7$ & $89.77(15)$ \\
$\mathrm{N} 2-\mathrm{Cu} 1-\mathrm{N} 4$ & $103.05(15)$ & $\mathrm{O} 51-\mathrm{Cu} 2-\mathrm{N} 8$ & $152.80(15)$ \\
$\mathrm{O} 53-\mathrm{Cu} 1-\mathrm{N} 1$ & $89.92(14)$ & $\mathrm{N} 5-\mathrm{Cu} 2-\mathrm{N} 8$ & $94.96(16)$ \\
$\mathrm{O} 53-\mathrm{Cu} 1-\mathrm{N} 3$ & $93.37(14)$ & $\mathrm{N} 7-\mathrm{Cu} 2-\mathrm{N} 8$ & $81.32(17)$ \\
$\mathrm{N} 1-\mathrm{Cu} 1-\mathrm{N} 2$ & $81.74(14)$ & $\mathrm{O} 51-\mathrm{Cu} 2-\mathrm{N} 6$ & $101.29(16)$ \\
$\mathrm{N} 3-\mathrm{Cu} 1-\mathrm{N} 2$ & $95.05(15)$ & $\mathrm{N} 5-\mathrm{Cu} 2-\mathrm{N} 6$ & $79.62(16)$ \\
$\mathrm{N} 1-\mathrm{Cu} 1-\mathrm{N} 4$ & $100.08(14)$ & $\mathrm{N} 7-\mathrm{Cu} 2-\mathrm{N} 6$ & $95.64(16)$ \\
$\mathrm{N} 3-\mathrm{Cu} 1-\mathrm{N} 4$ & $79.74(15)$ & $\mathrm{N} 8-\mathrm{Cu} 2-\mathrm{N} 6$ & $105.12(16)$ \\
\hline
\end{tabular}

\section{Supramolecular features}

In the crystal of (I), $\pi-\pi$ stacking interactions occur between neighbouring phen ligands and generate a two-dimensional supramolecular system in the (100) plane, as shown in Fig. 3. The N1- and N3 ${ }^{\text {vii }}$-phen [symmetry code: (vii) $x,-y+\frac{1}{2}, z+\frac{1}{2}$ ] ligands are nearly parallel, the dihedral angle being $5.00(11)^{\circ}$ and the shortest distance between the centroids of the aromatic rings (N1- and N3 ${ }^{\text {vii }}$-rings) being 3.647 (3) $\AA$. These findings indicate $\pi-\pi$ stacking between the N1- and N3 ${ }^{\text {vii }}$-phen ligands of neighbouring complexes. The same is true for the N5- and $\mathrm{C} 1^{\text {viii }}$-phen [symmetry code: (viii) $x,-y-\frac{1}{2}, z+\frac{1}{2}$ ] ligands, the dihedral angle being $8.48(13)^{\circ}$ and the shortest distance between the centroids of the aromatic rings (N5- and C41 ${ }^{\text {viii }}$-rings) being 3.671 (3) $\AA$.

The molecular packing of (I), as shown in Fig. 4, displays alternating layers along the $a$ axis, one layer consisting of

Table 2

Hydrogen-bond geometry $\left(\AA,{ }^{\circ}\right)$.

\begin{tabular}{|c|c|c|c|c|}
\hline$D-\mathrm{H} \cdots A$ & $D-\mathrm{H}$ & $\mathrm{H} \cdots A$ & $D \cdots A$ & $D-\mathrm{H} \cdots A$ \\
\hline $\mathrm{O} 1 W-\mathrm{H} 1 A \cdots \mathrm{O} 53$ & 0.97 & 1.90 & $2.819(5)$ & 157 \\
\hline $\mathrm{O} 1 W-\mathrm{H} 1 B \cdots \mathrm{O} 61^{\mathrm{i}}$ & 1.00 & 1.76 & $2.758(5)$ & 169 \\
\hline $\mathrm{O} 2 W-\mathrm{H} 2 A \cdots \mathrm{O} 61^{\mathrm{i}}$ & 0.97 & 1.75 & $2.708(5)$ & 169 \\
\hline $\mathrm{O} 2 W-\mathrm{H} 2 B \cdots \mathrm{O} 4 W^{\mathrm{ii}}$ & 0.83 & 2.04 & $2.843(6)$ & 160 \\
\hline $\mathrm{O} 3 W-\mathrm{H} 3 A \cdots \mathrm{O} 62^{\mathrm{iii}}$ & 1.00 & 1.69 & $2.685(5)$ & 172 \\
\hline $\mathrm{O} 3 W-\mathrm{H} 3 B \cdots \mathrm{O} 62$ & 1.00 & 1.84 & $2.731(7)$ & 147 \\
\hline $\mathrm{O} 4 W-\mathrm{H} 4 A \cdots \mathrm{O} 1 W$ & 1.01 & 1.79 & $2.756(6)$ & 160 \\
\hline $\mathrm{O} 4 W-\mathrm{H} 4 B \cdots \mathrm{O} 3 W$ & 0.86 & 1.94 & $2.750(7)$ & 157 \\
\hline $\mathrm{O} 5 W-\mathrm{H} 5 A \cdots \mathrm{O} 72$ & 0.96 & 1.87 & $2.818(8)$ & 169 \\
\hline $\mathrm{O} 5 W-\mathrm{H} 5 B \cdots \mathrm{O} 6 W^{\mathrm{iv}}$ & 0.97 & 1.84 & $2.779(7)$ & 164 \\
\hline $\mathrm{O} 73-\mathrm{H} 73 \cdots \mathrm{O} 8 \mathrm{~W}$ & 0.82 & 2.15 & $2.88(2)$ & 148 \\
\hline $\mathrm{O} 63-\mathrm{H} 63 \cdots \mathrm{O} 2 W$ & 0.82 & 1.85 & $2.638(5)$ & 161 \\
\hline $\mathrm{C} 3-\mathrm{H} 3 \cdots \mathrm{O} 1 W^{\mathrm{i}}$ & 0.93 & 2.59 & $3.240(6)$ & 127 \\
\hline $\mathrm{C} 8-\mathrm{H} 8 \cdots \mathrm{O} 6 \mathrm{~W}$ & 0.93 & 2.48 & $3.371(8)$ & 162 \\
\hline $\mathrm{C} 14-\mathrm{H} 14 \cdots \mathrm{O} 72$ & 0.93 & 2.50 & $3.388(10)$ & 159 \\
\hline $\mathrm{C} 21-\mathrm{H} 21 \cdots \mathrm{O} 4 W^{\mathrm{ii}}$ & 0.93 & 2.58 & $3.230(8)$ & 128 \\
\hline $\mathrm{C} 25-\mathrm{H} 25 \cdots \mathrm{O} 52$ & 0.93 & 2.47 & $3.033(6)$ & 119 \\
\hline $\mathrm{C} 33-\mathrm{H} 33 \cdots \mathrm{O} 62^{\mathrm{v}}$ & 0.93 & 2.55 & $3.247(8)$ & 132 \\
\hline $\mathrm{C} 38-\mathrm{H} 38 \cdots \mathrm{O} 61^{\mathrm{iii}}$ & 0.93 & 2.37 & $3.298(8)$ & 172 \\
\hline $\mathrm{C} 65-\mathrm{H} 65 \cdots \mathrm{O} 63^{\mathrm{vi}}$ & 0.93 & 2.49 & $3.410(6)$ & 172 \\
\hline $\mathrm{C} 73-\mathrm{H} 71 \cdots \mathrm{O} 7 W^{\text {iv }}$ & 0.93 & 2.58 & $3.413(13)$ & 149 \\
\hline
\end{tabular}

Symmetry codes: (i) $x,-y+\frac{1}{2}, z+\frac{1}{2}$; (ii) $-x+1, y+\frac{1}{2},-z+\frac{1}{2}$; (iii) $-x+1,-y,-z$; (iv) $x,-y+\frac{1}{2}, z-\frac{1}{2}$; (v) $-x+1, y-\frac{1}{2},-z+\frac{1}{2}$; (vi) $-x+1,-y+1,-z$. 
Figure 3

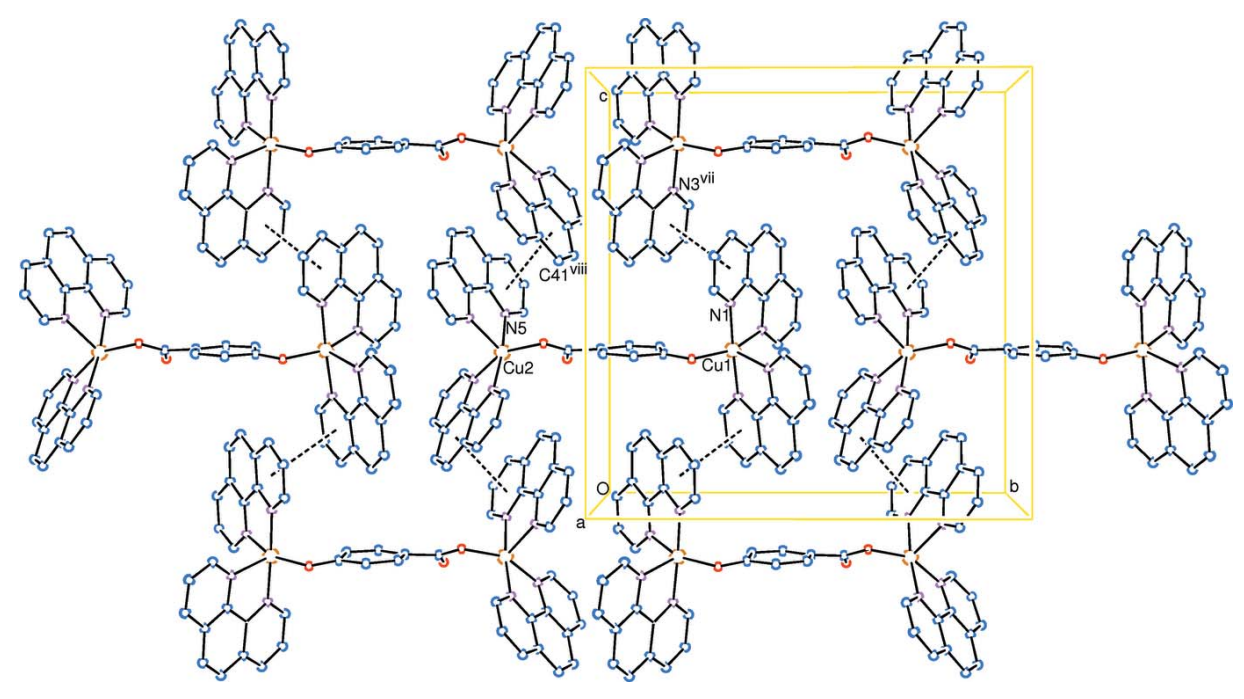

The $\pi-\pi$ stacking (dashed lines) between neighbouring dinuclear $\mathrm{Cu}^{\mathrm{II}}$ complex cations, forming a two-dimensional supramolecular system parallel to (100). $\mathrm{H}$ atoms have been omitted for clarity. [Symmetry codes: (vii) $x,-y+\frac{1}{2}, z+\frac{1}{2}$; (viii) $x,-y-\frac{1}{2}, z+\frac{1}{2}$ ]

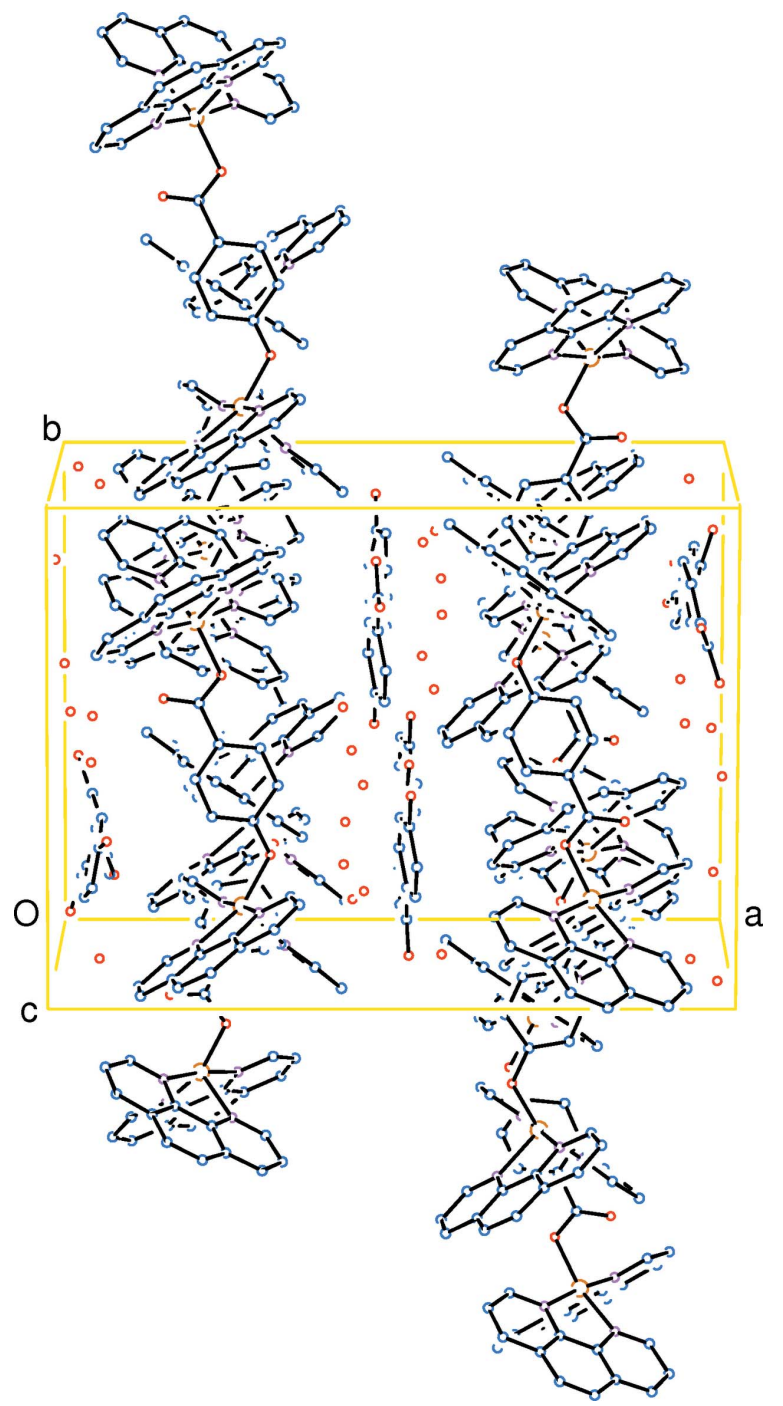

Figure 4

A packing diagram showing alternating layers along the $a$ axis, one layer consisting of dinuclear $\mathrm{Cu}^{\mathrm{II}}$ complex cations, the other consisting of noncoordinating 4-hydroxybenzoate dianions and solvent water molecules. $\mathrm{H}$ atoms have been omitted for clarity.

dinuclear $\mathrm{Cu}^{\mathrm{II}}$ complex cations (complex-layer), the other consisting of noncoordinating 4-hydroxybenzoate anions and solvent water molecules (solvent-layer). Abundant hydrogenbonding interactions occur within the solvent-layer and among the solvent- and complex-layers (Table 2). The $\mathrm{H}$ atoms on

Table 3

Experimental details.

Crystal data

Chemical formula

$M_{\mathrm{r}}$

Crystal system, space group

Temperature (K)

$a, b, c(\AA)$

$\beta\left({ }^{\circ}\right)$
$V\left(\AA^{3}\right)$

Radiation type

$\mu\left(\mathrm{mm}^{-1}\right)$

Crystal size (mm)

Data collection

Diffractometer

Absorption correction

$T_{\min }, T_{\max }$

No. of measured, independent and observed $[I>2 \sigma(I)]$ reflections

$R_{\text {int }}$

$(\sin \theta / \lambda)_{\max }\left(\AA^{-1}\right)$

Refinement

$R\left[F^{2}>2 \sigma\left(F^{2}\right)\right], w R\left(F^{2}\right), S$

No. of reflections

No. of parameters

$\mathrm{H}$-atom treatment

$\Delta \rho_{\max }, \Delta \rho_{\min }\left(\mathrm{e} \AA^{-3}\right)$

Computer programs: CrysAlis PRO (Agilent, 2014), SIR92 (Altomare et al., 1994), SHELXL2014 (Sheldrick, 2015) and ORTEP-3 for Windows and WinGX (Farrugia, 2012).

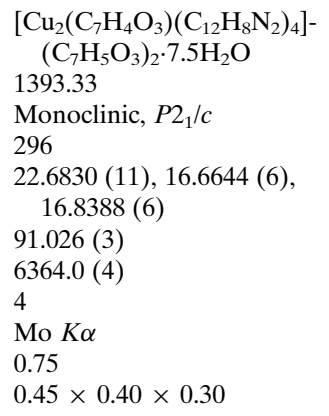

Xcalibur Atlas Gemini ultra Multi-scan (CrysAlis PRO; Agilent, 2014) $0.929,1.000$

27049, 11598, 7651

0.048

0.602

$0.067,0.201,1.03$
11598
861
H atoms treated by a mixture of
$\quad$ independent and constrained
$\quad$ refinement
$0.90,-0.56$

$0.067,0.201,1.03$ refinement $0.90,-0.56$ 
$\mathrm{O} 6 W, \mathrm{O} 7 W$ and $\mathrm{O} 8 W$ were not assigned in the structure, the separations $[\mathrm{O} 6 W \cdots \mathrm{O} 7 W=2.729(8) \AA$ and $\mathrm{O} 8 W \cdots \mathrm{O} 73$ 2.88 (2) $\AA$ ] suggest intermolecular hydrogen bonding between atoms $\mathrm{O} 6 \mathrm{~W}$ and $\mathrm{O} 7 \mathrm{~W}$, and between $\mathrm{O} 8 \mathrm{~W}$ and $\mathrm{O} 73$.

\section{Synthesis and crystallization}

Each reagent was available commercially and was of analytical grade. $\mathrm{CuCl}_{2} \cdot 2 \mathrm{H}_{2} \mathrm{O}(0.17 \mathrm{~g}, 1 \mathrm{mmol})$, 4-hydroxybenzoic acid $(0.28 \mathrm{~g}, 2 \mathrm{mmol}), 1,10$-phenanthroline $(0.20 \mathrm{~g}, 1 \mathrm{mmol})$ and $\mathrm{NaOH}(0.16 \mathrm{~g}, 4 \mathrm{mmol})$ were dissolved in $20 \mathrm{ml}$ water. The resulting solution was refluxed for $4 \mathrm{~h}$ and was then cooled to room temperature and filtered. Dark-green single crystals were obtained from the filtrate after five weeks.

\section{Refinement}

Crystal data, data collection and structure refinement details are summarized in Table 3. The disordered water $08 \mathrm{~W}$ atom was refined isotropically with a fixed occupacy of 0.5 . Aromatic and hydroxy $\mathrm{H}$ atoms were placed in calculated positions, with $\mathrm{C}-\mathrm{H}=0.93 \AA$ and $\mathrm{O}-\mathrm{H}=0.82 \AA$, and were included in the final cycles of refinement in riding mode, with
$U_{\text {iso }}(\mathrm{H})=1.2$ and $1.5 U_{\text {eq }}$ (parent), respectively. Water $\mathrm{H}$ atoms were located in difference Fourier map, and were refined with fixed positions and a fixed isotropic displacement parameter of $0.1 \AA^{2}$. The $\mathrm{H}$ atoms of the water molecules $\mathrm{O} 6 W, \mathrm{O} 7 W$ and O8W were not assigned. The peak corresponding to the maximum electron density in the difference Fourier map was close $(1.01 \AA)$ to atom O8W.

\section{Acknowledgements}

This project was supported by Startup fund for scientific research, Taizhou University.

\section{References}

Agilent (2014). CrysAlis PRO. Agilent Technologies Ltd, Yarnton, Oxfordshire, England.

Altomare, A., Cascarano, G., Giacovazzo, C., Guagliardi, A., Burla, M. C., Polidori, G. \& Camalli, M. (1994). J. Appl. Cryst. 27, 435.

Deisenhofer, J. \& Michel, H. (1989). EMBO J. 8, 2149-2170.

Farrugia, L. J. (2012). J. Appl. Cryst. 45, 849-854.

Sheldrick, G. M. (2015). Acta Cryst. A71, 3-8.

Su, J.-R., Gu, J.-M. \& Xu, D.-J. (2005). Acta Cryst. E61, m244-m246. 


\section{supporting information}

Acta Cryst. (2016). E72, 1396-1399 [https://doi.org/10.1107/S2056989016013943]

Crystal structure of $\mu$-4-oxidobenzoato- $\kappa^{2} O^{1}: O^{4}$-bis $[$ bis $(1,10$-phenanthroline$\left.\kappa^{2} N, N^{\prime}\right)$ copper(II)] bis(4-hydroxybenzoate) 7.5 -hydrate

\section{Jian-Rong Su and Yu Li}

Computing details

Data collection: CrysAlis PRO (Agilent, 2014); cell refinement: CrysAlis PRO (Agilent, 2014); data reduction: CrysAlis PRO (Agilent, 2014); program(s) used to solve structure: SIR92 (Altomare et al., 1994); program(s) used to refine structure: SHELXL2014 (Sheldrick, 2015); molecular graphics: ORTEP-3 for Windows (Farrugia, 2012); software used to prepare material for publication: $\operatorname{Win} G X$ (Farrugia, 2012).

$\mu$-4-Oxidobenzoato- $\kappa^{2} O^{1}: O^{4}$-bis [bis $\left(1,10\right.$-phenanthroline- $\left.\kappa^{2} N_{,} N^{\prime}\right)$ copper(II)] bis(4-hydroxybenzoate) 7.5-hydrate

Crystal data

$\left[\mathrm{Cu}_{2}\left(\mathrm{C}_{7} \mathrm{H}_{4} \mathrm{O}_{3}\right)\left(\mathrm{C}_{12} \mathrm{H}_{8} \mathrm{~N}_{2}\right)_{4}\right]\left(\mathrm{C}_{7} \mathrm{H}_{5} \mathrm{O}_{3}\right)_{2} \cdot 7.5 \mathrm{H}_{2} \mathrm{O}$

$M_{r}=1393.33$

Monoclinic, $P 2{ }_{1} / c$

$a=22.6830(11) \AA$

$b=16.6644(6) \AA$

$c=16.8388(6) \AA$

$\beta=91.026(3)^{\circ}$

$V=6364.0(4) \AA^{3}$

$Z=4$

\section{Data collection}

Xcalibur Atlas Gemini ultra diffractometer

Radiation source: Enhance (Mo) X-ray Source Detector resolution: 10.3592 pixels $\mathrm{mm}^{-1}$ $\omega$ scans

Absorption correction: multi-scan (CrysAlis PRO; Agilent, 2014)

$T_{\min }=0.929, T_{\max }=1.000$

\section{Refinement}

Refinement on $F^{2}$

Least-squares matrix: full

$R\left[F^{2}>2 \sigma\left(F^{2}\right)\right]=0.067$

$w R\left(F^{2}\right)=0.201$

$S=1.03$

11598 reflections

861 parameters

0 restraints
$F(000)=2884$

$D_{\mathrm{x}}=1.454 \mathrm{Mg} \mathrm{m}^{-3}$

Mo $K \alpha$ radiation, $\lambda=0.71073 \AA$

Cell parameters from 5425 reflections

$\theta=3.2-29.4^{\circ}$

$\mu=0.75 \mathrm{~mm}^{-1}$

$T=296 \mathrm{~K}$

Block, dark green

$0.45 \times 0.40 \times 0.30 \mathrm{~mm}$

27049 measured reflections

11598 independent reflections

7651 reflections with $I>2 \sigma(I)$

$R_{\text {int }}=0.048$

$\theta_{\max }=25.4^{\circ}, \theta_{\min }=3.2^{\circ}$

$h=-27 \rightarrow 24$

$k=-16 \rightarrow 20$

$l=-16 \rightarrow 20$

Hydrogen site location: mixed

$\mathrm{H}$ atoms treated by a mixture of independent and constrained refinement

$w=1 /\left[\sigma^{2}\left(F_{\mathrm{o}}^{2}\right)+(0.0916 P)^{2}+7.0203 P\right]$

where $P=\left(F_{\mathrm{o}}{ }^{2}+2 F_{\mathrm{c}}{ }^{2}\right) / 3$

$(\Delta / \sigma)_{\max }<0.001$

$\Delta \rho_{\max }=0.90 \mathrm{e} \AA^{-3}$

$\Delta \rho_{\min }=-0.56$ e $\AA^{-3}$ 


\section{Special details}

Experimental. Absorption correction: CrysAlis PRO, Agilent Technologies, Version 1.171.35.11 (release 16-05-2011 CrysAlis171 .NET) (compiled May 16 2011,17:55:39) Empirical absorption correction using spherical harmonics, implemented in SCALE3 ABSPACK scaling algorithm.

Geometry. All esds (except the esd in the dihedral angle between two 1.s. planes) are estimated using the full covariance matrix. The cell esds are taken into account individually in the estimation of esds in distances, angles and torsion angles; correlations between esds in cell parameters are only used when they are defined by crystal symmetry. An approximate (isotropic) treatment of cell esds is used for estimating esds involving l.s. planes.

Fractional atomic coordinates and isotropic or equivalent isotropic displacement parameters $\left(\AA^{2}\right)$

\begin{tabular}{|c|c|c|c|c|c|}
\hline & $x$ & $y$ & $z$ & $U_{\text {iso }} * / U_{\text {eq }}$ & Occ. $(<1)$ \\
\hline $\mathrm{Cu} 1$ & $0.28079(2)$ & $0.31771(3)$ & $0.36174(3)$ & $0.03912(18)$ & \\
\hline $\mathrm{Cu} 2$ & $0.21227(3)$ & $-0.25364(4)$ & $0.35545(3)$ & $0.04569(19)$ & \\
\hline $\mathrm{O} 51$ & $0.25057(16)$ & $-0.1490(2)$ & $0.3772(2)$ & $0.0555(9)$ & \\
\hline O52 & $0.16596(18)$ & $-0.0964(2)$ & $0.3379(2)$ & $0.0705(11)$ & \\
\hline O53 & 0.32159 (14) & $0.21783(19)$ & $0.34046(17)$ & $0.0449(8)$ & \\
\hline $\mathrm{C} 51$ & $0.2467(2)$ & $-0.0085(3)$ & $0.3632(3)$ & $0.0442(11)$ & \\
\hline $\mathrm{C} 52$ & $0.2127(2)$ & $0.0605(3)$ & $0.3706(3)$ & $0.0453(11)$ & \\
\hline H52 & 0.1723 & 0.0554 & 0.3776 & $0.054^{*}$ & \\
\hline $\mathrm{C} 53$ & $0.2368(2)$ & $0.1362(3)$ & $0.3678(2)$ & $0.0423(11)$ & \\
\hline H53 & 0.2130 & 0.1807 & 0.3762 & $0.051^{*}$ & \\
\hline $\mathrm{C} 54$ & $0.2969(2)$ & $0.1473(3)$ & $0.3527(2)$ & $0.0403(11)$ & \\
\hline $\mathrm{C} 55$ & $0.3326(2)$ & $0.0770(3)$ & $0.3476(3)$ & $0.0450(11)$ & \\
\hline $\mathrm{H} 55$ & 0.3729 & 0.0818 & 0.3403 & $0.054^{*}$ & \\
\hline $\mathrm{C} 56$ & $0.3070(2)$ & $0.0013(3)$ & $0.3535(3)$ & $0.0474(12)$ & \\
\hline H56 & 0.3310 & -0.0439 & 0.3509 & $0.057^{*}$ & \\
\hline $\mathrm{C} 57$ & $0.2179(2)$ & $-0.0904(3)$ & $0.3594(2)$ & $0.0468(12)$ & \\
\hline $\mathrm{N} 1$ & $0.30647(16)$ & $0.3111(2)$ & $0.4759(2)$ & $0.0388(9)$ & \\
\hline $\mathrm{N} 2$ & $0.21591(17)$ & $0.3878(2)$ & $0.4091(2)$ & $0.0425(9)$ & \\
\hline N3 & $0.25177(17)$ & $0.3299(2)$ & $0.2478(2)$ & $0.0416(9)$ & \\
\hline N4 & $0.34316(17)$ & $0.4048(2)$ & $0.3191(2)$ & $0.0409(9)$ & \\
\hline N5 & $0.15784(18)$ & $-0.2504(2)$ & $0.4483(2)$ & $0.0439(9)$ & \\
\hline N6 & $0.2601(2)$ & $-0.3322(2)$ & $0.4393(2)$ & $0.0531(11)$ & \\
\hline N7 & $0.26698(18)$ & $-0.2714(2)$ & $0.2643(2)$ & $0.0471(10)$ & \\
\hline N8 & $0.15934(19)$ & $-0.3302(2)$ & $0.2877(2)$ & $0.0475(10)$ & \\
\hline $\mathrm{C} 1$ & $0.3531(2)$ & $0.2736(3)$ & $0.5068(3)$ & $0.0452(11)$ & \\
\hline $\mathrm{H} 1$ & 0.3779 & 0.2456 & 0.4732 & $0.054^{*}$ & \\
\hline $\mathrm{C} 2$ & $0.3660(2)$ & $0.2747(3)$ & $0.5880(3)$ & $0.0480(12)$ & \\
\hline $\mathrm{H} 2$ & 0.3990 & 0.2477 & 0.6079 & $0.058^{*}$ & \\
\hline $\mathrm{C} 3$ & $0.3305(2)$ & 0.3153 & $0.6377(3)$ & $0.0517(13)$ & \\
\hline H3 & 0.3391 & 0.3161 & 0.6919 & $0.062^{*}$ & \\
\hline $\mathrm{C} 4$ & $0.2808(2)$ & 0.3563 & $0.6076(3)$ & $0.0454(11)$ & \\
\hline $\mathrm{C} 5$ & $0.2391(3)$ & 0.3988 & $0.6544(3)$ & $0.0596(15)$ & \\
\hline H5 & 0.2452 & 0.4020 & 0.7091 & $0.071^{*}$ & \\
\hline C6 & $0.1913(3)$ & $0.4342(3)$ & $0.6214(3)$ & $0.0579(14)$ & \\
\hline H6 & 0.1642 & 0.4593 & 0.6539 & $0.069^{*}$ & \\
\hline $\mathrm{C} 7$ & $0.1815(2)$ & $0.4341(3)$ & $0.5370(3)$ & $0.0477(12)$ & \\
\hline
\end{tabular}




\begin{tabular}{|c|c|c|c|c|}
\hline $\mathrm{C} 8$ & $0.1348(2)$ & 0.4741 & $0.4984(4)$ & $0.0622(15)$ \\
\hline H8 & 0.1073 & 0.5028 & 0.5273 & $0.075^{*}$ \\
\hline C9 & $0.1304(3)$ & $0.4703(4)$ & 0.4179 (4) & $0.0681(16)$ \\
\hline H9 & 0.0999 & 0.4970 & 0.3913 & $0.082 *$ \\
\hline $\mathrm{C} 10$ & $0.1714(2)$ & $0.4265(3)$ & $0.3746(3)$ & $0.0560(13)$ \\
\hline H10 & 0.1673 & 0.4244 & 0.3196 & $0.067^{*}$ \\
\hline $\mathrm{C} 11$ & $0.2211(2)$ & $0.3924(3)$ & $0.4898(3)$ & $0.0389(10)$ \\
\hline $\mathrm{C} 12$ & $0.2711(2)$ & $0.3523(3)$ & $0.5256(2)$ & $0.0380(10)$ \\
\hline $\mathrm{C} 13$ & $0.2056(2)$ & $0.2941(3)$ & $0.2141(3)$ & $0.0513(12)$ \\
\hline H13 & 0.1817 & 0.2621 & 0.2453 & $0.062^{*}$ \\
\hline $\mathrm{C} 14$ & $0.1915(3)$ & $0.3025(4)$ & $0.1335(3)$ & $0.0605(14)$ \\
\hline H14 & 0.1587 & 0.2767 & 0.1116 & $0.073^{*}$ \\
\hline $\mathrm{C} 15$ & $0.2262(3)$ & $0.3486(4)$ & $0.0876(3)$ & $0.0611(15)$ \\
\hline H15 & 0.2168 & 0.3551 & 0.0340 & $0.073^{*}$ \\
\hline $\mathrm{C} 16$ & $0.2755(2)$ & $0.3862(3)$ & $0.1196(3)$ & $0.0510(13)$ \\
\hline $\mathrm{C} 17$ & $0.3165(3)$ & $0.4348(4)$ & $0.0758(3)$ & $0.0648(16)$ \\
\hline $\mathrm{H} 17$ & 0.3104 & 0.4412 & 0.0214 & $0.078^{*}$ \\
\hline $\mathrm{C} 18$ & $0.3629(3)$ & 0.4709 (4) & $0.1108(3)$ & $0.0683(17)$ \\
\hline H18 & 0.3889 & 0.5004 & 0.0801 & $0.082 *$ \\
\hline C19 & $0.3732(2)$ & $0.4649(3)$ & $0.1948(3)$ & $0.0546(13)$ \\
\hline $\mathrm{C} 20$ & $0.4194(3)$ & $0.5043(3)$ & $0.2362(4)$ & $0.0666(16)$ \\
\hline $\mathrm{H} 20$ & 0.4452 & 0.5376 & 0.2093 & $0.080^{*}$ \\
\hline $\mathrm{C} 21$ & $0.4259(2)$ & $0.4930(3)$ & $0.3147(4)$ & $0.0627(15)$ \\
\hline $\mathrm{H} 21$ & 0.4561 & 0.5189 & 0.3426 & $0.075^{*}$ \\
\hline $\mathrm{C} 22$ & $0.3875(2)$ & $0.4429(3)$ & $0.3546(3)$ & $0.0509(12)$ \\
\hline $\mathrm{H} 22$ & 0.3933 & 0.4357 & 0.4089 & $0.061^{*}$ \\
\hline $\mathrm{C} 23$ & $0.3358(2)$ & $0.4162(3)$ & $0.2396(3)$ & $0.0416(11)$ \\
\hline $\mathrm{C} 24$ & $0.2863(2)$ & $0.3766(3)$ & $0.2021(2)$ & $0.0422(11)$ \\
\hline $\mathrm{C} 25$ & $0.1075(2)$ & $-0.2109(3)$ & $0.4523(3)$ & $0.0552(13)$ \\
\hline H25 & 0.0948 & -0.1815 & 0.4083 & $0.066^{*}$ \\
\hline $\mathrm{C} 26$ & $0.0724(3)$ & $-0.2114(4)$ & $0.5202(3)$ & $0.0637(15)$ \\
\hline $\mathrm{H} 26$ & 0.0372 & -0.1828 & 0.5208 & $0.076^{*}$ \\
\hline $\mathrm{C} 27$ & $0.0901(3)$ & $-0.2538(4)$ & $0.5846(3)$ & $0.0639(16)$ \\
\hline $\mathrm{H} 27$ & 0.0671 & -0.2548 & 0.6297 & $0.077^{*}$ \\
\hline $\mathrm{C} 28$ & $0.1432(3)$ & $-0.2959(3)$ & $0.5833(3)$ & $0.0558(14)$ \\
\hline $\mathrm{C} 29$ & $0.1664(3)$ & -0.3405 & $0.6497(3)$ & $0.0697(18)$ \\
\hline $\mathrm{H} 29$ & 0.1449 & -0.3437 & 0.6960 & $0.084^{*}$ \\
\hline $\mathrm{C} 30$ & $0.2190(4)$ & -0.3780 & $0.6459(3)$ & $0.0734(19)$ \\
\hline H30 & 0.2337 & -0.4047 & 0.6906 & $0.088^{*}$ \\
\hline $\mathrm{C} 31$ & $0.2526(3)$ & $-0.3777(3)$ & $0.5754(3)$ & $0.0567(14)$ \\
\hline $\mathrm{C} 32$ & $0.3070(3)$ & $-0.4161(4)$ & $0.5678(4)$ & $0.079(2)$ \\
\hline H32 & 0.3233 & -0.4443 & 0.6106 & $0.095^{*}$ \\
\hline $\mathrm{C} 33$ & $0.3362(3)$ & $-0.4122(4)$ & $0.4980(5)$ & $0.082(2)$ \\
\hline H33 & 0.3725 & -0.4374 & 0.4925 & $0.098^{*}$ \\
\hline $\mathrm{C} 34$ & $0.3107(3)$ & $-0.3700(4)$ & $0.4355(4)$ & $0.0733(17)$ \\
\hline H34 & 0.3307 & -0.3684 & 0.3878 & $0.088^{*}$ \\
\hline $\mathrm{C} 35$ & $0.2311(2)$ & $-0.3354(3)$ & $0.5093(3)$ & $0.0482(12)$ \\
\hline $\mathrm{C} 36$ & $0.1758(2)$ & -0.2933 & $0.5137(3)$ & $0.0470(12)$ \\
\hline
\end{tabular}




\begin{tabular}{|c|c|c|c|c|}
\hline $\mathrm{C} 37$ & $0.3202(2)$ & $-0.2411(3)$ & $0.2541(3)$ & $0.0568(14)$ \\
\hline H37 & 0.3362 & -0.2074 & 0.2929 & $0.068^{*}$ \\
\hline C38 & $0.3530(3)$ & $-0.2579(4)$ & $0.1875(4)$ & $0.0665(16)$ \\
\hline H38 & 0.3904 & -0.2359 & 0.1826 & $0.080^{*}$ \\
\hline C39 & $0.3308(3)$ & $-0.3062(4)$ & $0.1292(4)$ & $0.0655(15)$ \\
\hline H39 & 0.3530 & -0.3175 & 0.0847 & $0.079^{*}$ \\
\hline $\mathrm{C} 40$ & $0.2741(3)$ & $-0.3392(3)$ & $0.1368(3)$ & $0.0550(13)$ \\
\hline $\mathrm{C} 41$ & $0.2453(3)$ & $-0.3883(3)$ & $0.0788(3)$ & $0.0635(15)$ \\
\hline H41 & 0.2637 & -0.3984 & 0.0309 & $0.076^{*}$ \\
\hline $\mathrm{C} 42$ & $0.1923(3)$ & $-0.4201(4)$ & $0.0919(3)$ & $0.0692(17)$ \\
\hline $\mathrm{H} 42$ & 0.1755 & -0.4536 & 0.0536 & $0.083 *$ \\
\hline $\mathrm{C} 43$ & $0.1599(3)$ & $-0.4042(3)$ & $0.1637(3)$ & $0.0569(14)$ \\
\hline $\mathrm{C} 44$ & $0.1043(3)$ & $-0.4333(4)$ & $0.1801(4)$ & $0.0706(17)$ \\
\hline H44 & 0.0855 & -0.4680 & 0.1447 & $0.085^{*}$ \\
\hline $\mathrm{C} 45$ & $0.0771(3)$ & $-0.4111(4)$ & $0.2480(4)$ & $0.0736(18)$ \\
\hline H45 & 0.0397 & -0.4304 & 0.2594 & $0.088^{*}$ \\
\hline $\mathrm{C} 46$ & $0.1062(2)$ & $-0.3589(3)$ & $0.3002(3)$ & $0.0599(14)$ \\
\hline H46 & 0.0872 & -0.3436 & 0.3462 & $0.072^{*}$ \\
\hline C47 & $0.1866(2)$ & $-0.3534(3)$ & $0.2202(3)$ & $0.0461(11)$ \\
\hline $\mathrm{C} 48$ & $0.2441(2)$ & $-0.3208(3)$ & $0.2068(3)$ & $0.0470(12)$ \\
\hline O61 & $0.51842(17)$ & $0.1737(2)$ & $-0.1530(2)$ & $0.0586(9)$ \\
\hline O62 & $0.5244(2)$ & $0.0993(2)$ & $-0.0439(2)$ & $0.0754(12)$ \\
\hline O63 & $0.5268(2)$ & $0.4457(2)$ & $0.1098(2)$ & $0.0846(14)$ \\
\hline H63 & 0.5352 & 0.4351 & 0.1561 & $0.127^{*}$ \\
\hline C61 & $0.5215(2)$ & $0.2393(3)$ & $-0.0278(3)$ & $0.0455(11)$ \\
\hline C62 & $0.5337(3)$ & $0.2353(3)$ & $0.0539(3)$ & $0.0571(14)$ \\
\hline $\mathrm{H} 62$ & 0.5405 & 0.1854 & 0.0771 & $0.068^{*}$ \\
\hline C63 & $0.5359(3)$ & $0.3023(3)$ & $0.1005(3)$ & $0.0603(14)$ \\
\hline H63A & 0.5447 & 0.2980 & 0.1545 & $0.072^{*}$ \\
\hline C64 & $0.5248(3)$ & $0.3771(3)$ & $0.0665(3)$ & $0.0582(14)$ \\
\hline C65 & $0.5116(3)$ & $0.3817(3)$ & $-0.0132(3)$ & $0.0624(15)$ \\
\hline H65 & 0.5036 & 0.4314 & -0.0361 & $0.075^{*}$ \\
\hline C66 & $0.5101(3)$ & $0.3140(3)$ & $-0.0599(3)$ & $0.0553(13)$ \\
\hline H66 & 0.5012 & 0.3186 & -0.1138 & $0.066^{*}$ \\
\hline C67 & $0.5211(2)$ & $0.1660(3)$ & $-0.0794(3)$ & $0.0497(12)$ \\
\hline $\mathrm{O} 73$ & $0.0847(3)$ & $0.1727(5)$ & $0.4492(4)$ & $0.159(3)$ \\
\hline $\mathrm{H} 73$ & 0.0901 & 0.1240 & 0.4488 & $0.238^{*}$ \\
\hline O71 & $0.0240(3)$ & 0.3687 (4) & 0.1367 (4) & $0.135(2)$ \\
\hline $\mathrm{O} 72$ & $0.0528(3)$ & $0.2498(4)$ & $0.0767(5)$ & $0.125(2)$ \\
\hline C71 & $0.0508(3)$ & $0.2611(5)$ & $0.2203(7)$ & $0.104(3)$ \\
\hline $\mathrm{C} 72$ & 0.0645 (4) & $0.1836(6)$ & $0.2302(6)$ & 0.110 \\
\hline H72 & 0.0674 & 0.1508 & 0.1858 & $0.132 *$ \\
\hline $\mathrm{C} 73$ & 0.0747 (4) & $0.1501(6)$ & $0.3063(6)$ & 0.121 \\
\hline $\mathrm{H} 71$ & 0.0830 & 0.0956 & 0.3114 & $0.145^{*}$ \\
\hline $\mathrm{C} 74$ & $0.0723(4)$ & $0.1985(6)$ & $0.3739(7)$ & 0.110 \\
\hline $\mathrm{C} 75$ & $0.0613(4)$ & $0.2808(7)$ & $0.3649(7)$ & 0.118 \\
\hline H75 & 0.0622 & 0.3146 & 0.4089 & $0.141^{*}$ \\
\hline C76 & $0.0485(4)$ & $0.3131(7)$ & $0.2862(6)$ & $0.115(3)$ \\
\hline
\end{tabular}




\begin{tabular}{|c|c|c|c|c|c|}
\hline $\mathrm{H} 76$ & 0.0390 & 0.3670 & 0.2795 & $0.138^{*}$ & \\
\hline $\mathrm{C} 77$ & $0.0414(4)$ & $0.2948(7)$ & $0.1392(6)$ & $0.109(3)$ & \\
\hline $\mathrm{O} 1 \mathrm{~W}$ & $0.42849(17)$ & $0.2505(2)$ & $0.2640(2)$ & $0.0635(10)$ & \\
\hline $\mathrm{O} 2 \mathrm{~W}$ & $0.5730(2)$ & $0.4352(2)$ & $0.2543(2)$ & $0.0740(11)$ & \\
\hline $\mathrm{O} 3 \mathrm{~W}$ & $0.4390(2)$ & $0.0531(2)$ & 0.0588 (3) & $0.0781(12)$ & \\
\hline $\mathrm{O} 4 \mathrm{~W}$ & $0.4567(2)$ & $0.0978(2)$ & $0.2146(2)$ & $0.0856(14)$ & \\
\hline $\mathrm{O} 5 \mathrm{~W}$ & $-0.0116(2)$ & $0.1084(3)$ & $0.0453(3)$ & $0.0922(14)$ & \\
\hline O6W & $0.0242(2)$ & $0.5351(3)$ & $0.6151(3)$ & $0.1036(16)$ & \\
\hline $\mathrm{O} 7 \mathrm{~W}$ & $0.0604(3)$ & $0.5505(5)$ & 0.7698 (4) & $0.153(3)$ & \\
\hline O8W & $0.0634(10)$ & $0.0074(14)$ & $0.4893(13)$ & $0.227(8)^{*}$ & 0.5 \\
\hline $\mathrm{H} 1 \mathrm{~A}$ & 0.3987 & 0.2333 & 0.3013 & $0.100 *$ & \\
\hline H1B & 0.4602 & 0.2736 & 0.2996 & $0.100 *$ & \\
\hline $\mathrm{H} 2 \mathrm{~A}$ & 0.5517 & 0.4018 & 0.2909 & $0.100 *$ & \\
\hline $\mathrm{H} 2 \mathrm{~B}$ & 0.5714 & 0.4851 & 0.2567 & $0.100 *$ & \\
\hline $\mathrm{H} 3 \mathrm{~A}$ & 0.4549 & -0.0030 & 0.0576 & $0.100 *$ & \\
\hline H3B & 0.4725 & 0.0848 & 0.0377 & $0.100 *$ & \\
\hline $\mathrm{H} 4 \mathrm{~A}$ & 0.4564 & 0.1556 & 0.2317 & $0.100^{*}$ & \\
\hline H4B & 0.4475 & 0.0970 & 0.1650 & $0.100 *$ & \\
\hline $\mathrm{H} 5 \mathrm{~A}$ & 0.0137 & 0.1524 & 0.0598 & $0.100 *$ & \\
\hline H5B & 0.0042 & 0.0564 & 0.0602 & $0.100 *$ & \\
\hline
\end{tabular}

Atomic displacement parameters $\left(\AA^{2}\right)$

\begin{tabular}{lllllll}
\hline & $U^{11}$ & $U^{22}$ & $U^{33}$ & $U^{12}$ & $U^{13}$ & $U^{23}$ \\
\hline Cu1 & $0.0415(3)$ & $0.0474(3)$ & $0.0285(3)$ & $0.0009(2)$ & $-0.0005(2)$ & $-0.0004(2)$ \\
Cu2 & $0.0418(4)$ & $0.0512(4)$ & $0.0442(3)$ & $-0.0054(3)$ & $0.0042(3)$ & $0.0018(3)$ \\
O51 & $0.054(2)$ & $0.053(2)$ & $0.059(2)$ & $-0.0086(18)$ & $0.0087(17)$ & $-0.0072(18)$ \\
O52 & $0.061(3)$ & $0.074(3)$ & $0.076(3)$ & $-0.020(2)$ & $-0.023(2)$ & $0.018(2)$ \\
O53 & $0.049(2)$ & $0.0449(18)$ & $0.0413(17)$ & $-0.0024(15)$ & $0.0074(14)$ & $-0.0023(15)$ \\
C51 & $0.052(3)$ & $0.046(3)$ & $0.034(2)$ & $-0.006(2)$ & $0.004(2)$ & $0.000(2)$ \\
C52 & $0.039(3)$ & $0.060(3)$ & $0.038(2)$ & $-0.002(2)$ & $0.005(2)$ & $0.002(2)$ \\
C53 & $0.044(3)$ & $0.049(3)$ & $0.035(2)$ & $0.001(2)$ & $0.0104(19)$ & $-0.002(2)$ \\
C54 & $0.052(3)$ & $0.048(3)$ & $0.0215(19)$ & $-0.014(2)$ & $-0.0012(18)$ & $-0.0006(19)$ \\
C55 & $0.037(3)$ & $0.050(3)$ & $0.047(3)$ & $-0.003(2)$ & $0.009(2)$ & $-0.006(2)$ \\
C56 & $0.050(3)$ & $0.047(3)$ & $0.045(3)$ & $-0.001(2)$ & $0.006(2)$ & $-0.006(2)$ \\
C57 & $0.048(3)$ & $0.070(3)$ & $0.023(2)$ & $-0.001(3)$ & $0.004(2)$ & $0.001(2)$ \\
N1 & $0.037(2)$ & $0.049(2)$ & $0.0297(18)$ & $0.0019(17)$ & $-0.0010(15)$ & $-0.0002(16)$ \\
N2 & $0.040(2)$ & $0.053(2)$ & $0.0348(19)$ & $0.0025(18)$ & $-0.0009(16)$ & $0.0042(18)$ \\
N3 & $0.041(2)$ & $0.054(2)$ & $0.0300(18)$ & $0.0006(18)$ & $0.0007(16)$ & $-0.0032(17)$ \\
N4 & $0.043(2)$ & $0.045(2)$ & $0.0343(19)$ & $-0.0016(18)$ & $-0.0009(16)$ & $0.0006(17)$ \\
N5 & $0.038(2)$ & $0.050(2)$ & $0.044(2)$ & $-0.0034(18)$ & $0.0007(17)$ & $0.0018(18)$ \\
N6 & $0.053(3)$ & $0.053(2)$ & $0.053(2)$ & $0.005(2)$ & $-0.001(2)$ & $0.004(2)$ \\
N7 & $0.045(2)$ & $0.052(2)$ & $0.045(2)$ & $-0.0036(19)$ & $0.0054(18)$ & $0.0035(19)$ \\
N8 & $0.049(3)$ & $0.046(2)$ & $0.048(2)$ & $-0.0048(19)$ & $-0.0017(19)$ & $0.0075(19)$ \\
C1 & $0.042(3)$ & $0.048(3)$ & $0.045(3)$ & $0.004(2)$ & $-0.002(2)$ & $0.001(2)$ \\
C2 & $0.041(3)$ & $0.056(3)$ & $0.047(3)$ & $-0.002(2)$ & $-0.009(2)$ & $0.007(2)$ \\
C3 & $0.059(3)$ & $0.064(3)$ & $0.032(2)$ & $-0.009(3)$ & $-0.007(2)$ & $0.008(2)$ \\
C4 & $0.055(3)$ & $0.048(3)$ & $0.033(2)$ & $-0.008(2)$ & $0.000(2)$ & $0.004(2)$
\end{tabular}




\begin{tabular}{|c|c|c|c|c|c|c|}
\hline $\mathrm{C} 5$ & $0.085(4)$ & $0.063(3)$ & $0.031(2)$ & $0.000(3)$ & $0.012(3)$ & $-0.005(2)$ \\
\hline C6 & $0.067(4)$ & $0.054(3)$ & $0.053(3)$ & $0.006(3)$ & $0.023(3)$ & -0.009 (3) \\
\hline $\mathrm{C} 7$ & $0.048(3)$ & $0.048(3)$ & $0.048(3)$ & $0.000(2)$ & $0.012(2)$ & $0.001(2)$ \\
\hline $\mathrm{C} 8$ & $0.050(3)$ & $0.058(3)$ & $0.079(4)$ & $0.011(3)$ & $0.011(3)$ & $-0.004(3)$ \\
\hline C9 & $0.051(4)$ & $0.069(4)$ & $0.085(4)$ & $0.019(3)$ & $-0.008(3)$ & $0.002(3)$ \\
\hline $\mathrm{C} 10$ & $0.052(3)$ & $0.066(3)$ & $0.050(3)$ & $0.004(3)$ & $-0.008(2)$ & $0.005(3)$ \\
\hline $\mathrm{C} 11$ & $0.039(3)$ & $0.039(2)$ & $0.039(2)$ & $-0.003(2)$ & 0.0034 (19) & $-0.003(2)$ \\
\hline $\mathrm{C} 12$ & $0.040(3)$ & $0.040(2)$ & $0.034(2)$ & $-0.005(2)$ & 0.0043 (19) & $-0.003(2)$ \\
\hline $\mathrm{C} 13$ & $0.051(3)$ & $0.061(3)$ & $0.041(3)$ & -0.008 & $-0.005(2)$ & $-0.006(2)$ \\
\hline $\mathrm{C} 14$ & $0.057(4)$ & 0.077 (4) & $0.047(3)$ & 0.007 (3) & $-0.013(3)$ & $-0.011(3)$ \\
\hline $\mathrm{C} 15$ & $0.071(4)$ & $0.079(4)$ & $0.033(3)$ & $0.016(3)$ & $-0.015(3)$ & $-0.005(3)$ \\
\hline $\mathrm{C} 16$ & $0.058(3)$ & $0.060(3)$ & $0.035(2)$ & $0.016(3)$ & $0.005(2)$ & $0.001(2)$ \\
\hline $\mathrm{C} 17$ & $0.085(5)$ & $0.071(4)$ & $0.039(3)$ & $0.023(3)$ & $0.010(3)$ & $0.020(3)$ \\
\hline C18 & $0.074(4)$ & $0.068(4)$ & $0.064(4)$ & 0.007 (3) & $0.026(3)$ & $0.030(3)$ \\
\hline C19 & $0.052(3)$ & $0.049(3)$ & 0.063 & $0.007(2)$ & $0.010(3)$ & $0.019(3)$ \\
\hline $\mathrm{C} 20$ & $0.047(3)$ & $0.060(3)$ & $0.093(5)$ & $-0.006(3)$ & $0.018(3)$ & $0.024(3)$ \\
\hline $\mathrm{C} 21$ & $0.045(3)$ & $0.063(3)$ & $0.080(4)$ & -0.010 & -0.001 & $0.005(3)$ \\
\hline $\mathrm{C} 22$ & $0.046(3)$ & $0.053(3)$ & $0.053(3)$ & $0.000(2)$ & $-0.005(2)$ & $0.000(2)$ \\
\hline $\mathrm{C} 23$ & $0.040(3)$ & $0.040(2)$ & $0.044(3)$ & $0.008(2)$ & $0.005(2)$ & $0.008(2)$ \\
\hline $\mathrm{C} 24$ & $0.050(3)$ & $0.045(3)$ & $0.032(2)$ & $0.012(2)$ & $0.002(2)$ & $0.001(2)$ \\
\hline $\mathrm{C} 25$ & $0.045(3)$ & $0.066(3)$ & $0.056(3)$ & $-0.005(3)$ & $-0.001(2)$ & $0.004(3)$ \\
\hline $\mathrm{C} 26$ & $0.047(3)$ & $0.082(4)$ & $0.062(4)$ & $-0.008(3)$ & $0.007(3)$ & $-0.008(3)$ \\
\hline $\mathrm{C} 27$ & $0.061(4)$ & $0.078(4)$ & $0.053(3)$ & $-0.025(3)$ & $0.018(3)$ & $-0.013(3)$ \\
\hline $\mathrm{C} 28$ & $0.073(4)$ & $0.056(3)$ & 0.038 & $-0.021(3)$ & $0.002(2)$ & $-0.007(2)$ \\
\hline $\mathrm{C} 29$ & $0.115(6)$ & $0.058(3)$ & $0.037(3)$ & -0.019 (4) & 0.007 (3) & $-0.002(3)$ \\
\hline C30 & $0.125(6)$ & $0.053(3)$ & $0.042(3)$ & $-0.001(4)$ & $-0.018(3)$ & -0.002 \\
\hline C31 & $0.081(4)$ & $0.042(3)$ & $0.046(3)$ & $-0.007(3)$ & $-0.018(3)$ & $0.001(2)$ \\
\hline C32 & $0.101(6)$ & $0.062(4)$ & $0.074(4)$ & $0.006(4)$ & -0.038 & $0.003(3)$ \\
\hline C33 & $0.069(4)$ & 0.077 (4) & $0.098(5)$ & $0.023(4)$ & $-0.026(4)$ & $0.002(4)$ \\
\hline C34 & $0.058(4)$ & $0.076(4)$ & $0.085(4)$ & $0.010(3)$ & -0.005 & $0.010(3)$ \\
\hline $\mathrm{C} 35$ & $0.053(3)$ & $0.041(3)$ & $0.050(3)$ & $-0.007(2)$ & $-0.010(2)$ & $-0.004(2)$ \\
\hline $\mathrm{C} 36$ & $0.058(3)$ & $0.045(3)$ & $0.038(2)$ & $-0.013(2)$ & $0.001(2)$ & $-0.001(2)$ \\
\hline C37 & $0.043(3)$ & $0.065(3)$ & $0.062(3)$ & $-0.009(3)$ & $0.004(3)$ & $-0.001(3)$ \\
\hline C38 & $0.051(4)$ & 0.077 (4) & $0.072(4)$ & $-0.003(3)$ & $0.016(3)$ & $0.008(3)$ \\
\hline C39 & $0.062(4)$ & $0.068(4)$ & $0.067(4)$ & $0.009(3)$ & $0.021(3)$ & $0.006(3)$ \\
\hline $\mathrm{C} 40$ & $0.058(4)$ & $0.057(3)$ & 0.050 & $0.015(3)$ & $0.008(2)$ & $0.002(3)$ \\
\hline $\mathrm{C} 41$ & $0.075(4)$ & $0.062(3)$ & $0.054(3)$ & $0.012(3)$ & $0.005(3)$ & $-0.003(3)$ \\
\hline $\mathrm{C} 42$ & $0.088(5)$ & $0.068(4)$ & $0.052(3)$ & 0.009 (3) & $-0.013(3)$ & $-0.011(3)$ \\
\hline $\mathrm{C} 43$ & $0.059(4)$ & $0.049(3)$ & $0.062(3)$ & $0.002(3)$ & $-0.015(3)$ & $0.000(3)$ \\
\hline $\mathrm{C} 44$ & $0.067(4)$ & $0.072(4)$ & $0.072(4)$ & $-0.014(3)$ & $-0.017(3)$ & $-0.007(3)$ \\
\hline $\mathrm{C} 45$ & $0.056(4)$ & $0.077(4)$ & $0.086(4)$ & $-0.027(3)$ & $-0.011(3)$ & $0.008(4)$ \\
\hline $\mathrm{C} 46$ & $0.049(3)$ & $0.070(4)$ & $0.061(3)$ & -0.010 & $0.005(3)$ & $0.009(3)$ \\
\hline $\mathrm{C} 47$ & $0.052(3)$ & $0.042(3)$ & $0.044(3)$ & $0.002(2)$ & $-0.005(2)$ & $0.006(2)$ \\
\hline $\mathrm{C} 48$ & $0.054(3)$ & $0.041(3)$ & $0.046(3)$ & $0.005(2)$ & $-0.002(2)$ & 0.007 (2) \\
\hline O61 & $0.064(3)$ & $0.052(2)$ & $0.059(2)$ & $0.0041(18)$ & $-0.0006(18)$ & -0.0072 \\
\hline O62 & $0.106(4)$ & $0.038(2)$ & $0.083(3)$ & $0.005(2)$ & $0.003(2)$ & $0.002(2)$ \\
\hline O63 & $0.138(4)$ & $0.050(2)$ & $0.066(3)$ & $0.016(2)$ & $-0.007(2)$ & $-0.010(2)$ \\
\hline C61 & $0.041(3)$ & $0.041(3)$ & $0.055(3)$ & $0.000(2)$ & $0.005(2)$ & $-0.002(2)$ \\
\hline
\end{tabular}




\begin{tabular}{|c|c|c|c|c|c|c|}
\hline C62 & $0.068(4)$ & $0.038(3)$ & $0.065(3)$ & $0.000(2)$ & $0.001(3)$ & $0.005(3)$ \\
\hline C63 & $0.072(4)$ & $0.060(3)$ & $0.048(3)$ & $0.002(3)$ & $-0.003(3)$ & $0.003(3)$ \\
\hline C64 & $0.077(4)$ & $0.042(3)$ & $0.056(3)$ & $0.007(3)$ & $0.004(3)$ & -0.001 \\
\hline C65 & $0.091(5)$ & $0.040(3)$ & $0.057(3)$ & $0.006(3)$ & $0.000(3)$ & $-0.003(3)$ \\
\hline C66 & $0.068(4)$ & $0.047(3)$ & $0.051(3)$ & $0.003(3)$ & $0.000(3)$ & $0.007(2)$ \\
\hline C67 & $0.041(3)$ & $0.041(3)$ & $0.067(4)$ & $0.001(2)$ & $0.003(2)$ & $0.004(3)$ \\
\hline $\mathrm{O} 73$ & $0.125(6)$ & $0.246(9)$ & $0.106(5)$ & $0.083(6)$ & $0.012(4)$ & $0.027(5)$ \\
\hline O71 & $0.131(6)$ & $0.113(5)$ & $0.160(6)$ & $-0.001(4)$ & -0.008 & $0.019(4)$ \\
\hline $\mathrm{O} 72$ & $0.098(5)$ & $0.127(5)$ & $0.148(6)$ & $-0.019(4)$ & $-0.044(4)$ & $-0.001(4)$ \\
\hline C71 & $0.056(5)$ & $0.095(6)$ & $0.160(9)$ & $-0.008(4)$ & $-0.017(5)$ & $0.029(6)$ \\
\hline $\mathrm{C} 72$ & 0.087 (6) & $0.109(7)$ & $0.135(8)$ & $-0.004(5)$ & $-0.017(5)$ & $-0.013(6)$ \\
\hline C73 & $0.102(7)$ & $0.127(7)$ & $0.132(8)$ & $0.027(6)$ & $-0.026(6)$ & $-0.043(7)$ \\
\hline C74 & $0.078(6)$ & $0.115(7)$ & $0.137(8)$ & $0.009(5)$ & $0.006(5)$ & $-0.001(7)$ \\
\hline $\mathrm{C} 75$ & $0.075(6)$ & $0.131(8)$ & $0.147(9)$ & $-0.004(5)$ & $0.019(5)$ & $-0.037(7)$ \\
\hline C76 & $0.088(6)$ & $0.154(9)$ & $0.103(7)$ & $-0.018(6)$ & $0.027(5)$ & $-0.010(7)$ \\
\hline C77 & $0.066(5)$ & $0.133(8)$ & $0.128(8)$ & $-0.020(5)$ & $-0.019(5)$ & $0.015(7)$ \\
\hline $\mathrm{O} 1 \mathrm{~W}$ & $0.055(2)$ & 0.078 & $0.058(2)$ & $-0.0063(19)$ & $0.0042(18)$ & $-0.0151(19)$ \\
\hline $\mathrm{O} 2 \mathrm{~W}$ & $0.090(3)$ & $0.066(2)$ & $0.066(2)$ & $-0.008(2)$ & $0.017(2)$ & $0.001(2)$ \\
\hline $\mathrm{O} 3 \mathrm{~W}$ & $0.089(3)$ & $0.055(2)$ & $0.089(3)$ & 0.007 (2) & $0.001(2)$ & $-0.011(2)$ \\
\hline O4W & $0.112(4)$ & $0.069(3)$ & $0.075(3)$ & $0.035(3)$ & 0.009 (3) & $0.002(2)$ \\
\hline O5W & $0.079(3)$ & $0.077(3)$ & $0.120(4)$ & $-0.003(2)$ & $0.010(3)$ & $-0.004(3)$ \\
\hline O6W & $0.082(4)$ & $0.095(3)$ & $0.134(4)$ & $0.008(3)$ & $-0.004(3)$ & -0.020 \\
\hline O7W & $0.136(6)$ & $0.208(7)$ & $0.115(5)$ & $-0.034(5)$ & -0.030 & $-0.005(5)$ \\
\hline
\end{tabular}

Geometric parameters (A, $\stackrel{\circ}{)}$

\begin{tabular}{|c|c|c|c|}
\hline $\mathrm{Cu} 1-\mathrm{O} 53$ & $1.941(3)$ & $\mathrm{C} 25-\mathrm{C} 26$ & $1.406(8)$ \\
\hline $\mathrm{Cu} 1-\mathrm{N} 1$ & $2.002(3)$ & $\mathrm{C} 25-\mathrm{H} 25$ & 0.9300 \\
\hline $\mathrm{Cu} 1-\mathrm{N} 2$ & $2.051(4)$ & $\mathrm{C} 26-\mathrm{C} 27$ & $1.349(8)$ \\
\hline $\mathrm{Cu} 1-\mathrm{N} 3$ & $2.027(3)$ & $\mathrm{C} 26-\mathrm{H} 26$ & 0.9300 \\
\hline $\mathrm{Cu} 1-\mathrm{N} 4$ & $2.158(4)$ & $\mathrm{C} 27-\mathrm{C} 28$ & $1.394(8)$ \\
\hline $\mathrm{Cu} 2-\mathrm{O} 51$ & $1.979(3)$ & $\mathrm{C} 27-\mathrm{H} 27$ & 0.9300 \\
\hline $\mathrm{Cu} 2-\mathrm{N} 5$ & $2.010(4)$ & $\mathrm{C} 28-\mathrm{C} 36$ & $1.399(7)$ \\
\hline $\mathrm{Cu} 2-\mathrm{N} 6$ & $2.197(4)$ & C28-C29 & $1.435(8)$ \\
\hline $\mathrm{Cu} 2-\mathrm{N} 7$ & $2.013(4)$ & C29-C30 & $1.349(9)$ \\
\hline $\mathrm{Cu} 2-\mathrm{N} 8$ & $2.079(4)$ & С29- & 0.9300 \\
\hline $\mathrm{O} 51-\mathrm{C} 57$ & $1.259(6)$ & $\mathrm{C} 30-\mathrm{C} 31$ & $1.422(9)$ \\
\hline $\mathrm{O} 52-\mathrm{C} 57$ & $1.230(6)$ & C30- $\mathrm{H} 30$ & 0.9300 \\
\hline $\mathrm{O} 53-\mathrm{C} 54$ & $1.320(5)$ & C31-C35 & $1.397(7)$ \\
\hline C51-C52 & $1.392(7)$ & C31-C32 & $1.399(9)$ \\
\hline C51-C56 & $1.392(7)$ & C32-C33 & $1.361(10)$ \\
\hline C51-C57 & $1.514(7)$ & C32-H32 & 0.9300 \\
\hline C52-C53 & $1.375(7)$ & C33-C34 & $1.384(8)$ \\
\hline С $52-\mathrm{H} 52$ & 0.9300 & C33-H33 & 0.9300 \\
\hline C53-C54 & $1.402(7)$ & C34-H34 & 0.9300 \\
\hline C53-H53 & 0.9300 & C35-C36 & $1.439(7)$ \\
\hline $\mathrm{C} 54-\mathrm{C} 55$ & $1.427(7)$ & C37-C38 & $1.384(8)$ \\
\hline C55-C56 & $1.393(7)$ & C37-H37 & 0.9300 \\
\hline
\end{tabular}




\begin{tabular}{|c|c|c|c|}
\hline C55-H55 & 0.9300 & $\mathrm{C} 38-\mathrm{C} 39$ & $1.359(8)$ \\
\hline C56-H56 & 0.9300 & C $38-\mathrm{H} 38$ & 0.9300 \\
\hline $\mathrm{N} 1-\mathrm{C} 1$ & $1.327(6)$ & $\mathrm{C} 39-\mathrm{C} 40$ & $1.406(8)$ \\
\hline $\mathrm{N} 1-\mathrm{C} 12$ & $1.356(6)$ & C39-H39 & 0.9300 \\
\hline $\mathrm{N} 2-\mathrm{C} 10$ & $1.323(6)$ & $\mathrm{C} 40-\mathrm{C} 48$ & $1.405(7)$ \\
\hline $\mathrm{N} 2-\mathrm{C} 11$ & $1.363(5)$ & $\mathrm{C} 40-\mathrm{C} 41$ & $1.423(8)$ \\
\hline $\mathrm{N} 3-\mathrm{C} 13$ & $1.324(6)$ & $\mathrm{C} 41-\mathrm{C} 42$ & $1.336(9)$ \\
\hline N3-C24 & $1.354(6)$ & $\mathrm{C} 41-\mathrm{H} 41$ & 0.9300 \\
\hline $\mathrm{N} 4-\mathrm{C} 22$ & $1.324(6)$ & $\mathrm{C} 42-\mathrm{C} 43$ & $1.450(8)$ \\
\hline $\mathrm{N} 4-\mathrm{C} 23$ & $1.361(5)$ & $\mathrm{C} 42-\mathrm{H} 42$ & 0.9300 \\
\hline N5-C25 & $1.320(6)$ & $\mathrm{C} 43-\mathrm{C} 44$ & $1.384(8)$ \\
\hline N5-C36 & $1.369(6)$ & $\mathrm{C} 43-\mathrm{C} 47$ & $1.403(7)$ \\
\hline N6-C34 & $1.313(7)$ & $\mathrm{C} 44-\mathrm{C} 45$ & $1.359(9)$ \\
\hline N6-C35 & $1.362(7)$ & $\mathrm{C} 44-\mathrm{H} 44$ & 0.9300 \\
\hline N7-C37 & $1.323(6)$ & $\mathrm{C} 45-\mathrm{C} 46$ & $1.395(8)$ \\
\hline $\mathrm{N} 7-\mathrm{C} 48$ & $1.367(6)$ & $\mathrm{C} 45-\mathrm{H} 45$ & 0.9300 \\
\hline $\mathrm{N} 8-\mathrm{C} 46$ & $1.316(6)$ & $\mathrm{C} 46-\mathrm{H} 46$ & 0.9300 \\
\hline $\mathrm{N} 8-\mathrm{C} 47$ & $1.360(6)$ & $\mathrm{C} 47-\mathrm{C} 48$ & $1.434(7)$ \\
\hline $\mathrm{C} 1-\mathrm{C} 2$ & $1.393(6)$ & O61-C67 & $1.246(6)$ \\
\hline $\mathrm{C} 1-\mathrm{H} 1$ & 0.9300 & $\mathrm{O} 62-\mathrm{C} 67$ & $1.263(6)$ \\
\hline $\mathrm{C} 2-\mathrm{C} 3$ & $1.354(7)$ & $\mathrm{O} 63-\mathrm{C} 64$ & $1.357(6)$ \\
\hline $\mathrm{C} 2-\mathrm{H} 2$ & 0.9300 & $\mathrm{O} 63-\mathrm{H} 63$ & 0.8200 \\
\hline $\mathrm{C} 3-\mathrm{C} 4$ & $1.406(7)$ & C61-C66 & $1.379(7)$ \\
\hline $\mathrm{C} 3-\mathrm{H} 3$ & 0.9300 & C61-C62 & $1.399(7)$ \\
\hline $\mathrm{C} 4-\mathrm{C} 12$ & $1.396(6)$ & C61-C67 & $1.500(7)$ \\
\hline $\mathrm{C} 4-\mathrm{C} 5$ & $1.430(7)$ & $\mathrm{C} 62-\mathrm{C} 63$ & $1.365(7)$ \\
\hline $\mathrm{C} 5-\mathrm{C} 6$ & $1.345(8)$ & C62-H62 & 0.9300 \\
\hline $\mathrm{C} 5-\mathrm{H} 5$ & 0.9300 & $\mathrm{C} 63-\mathrm{C} 64$ & $1.392(7)$ \\
\hline $\mathrm{C} 6-\mathrm{C} 7$ & $1.436(7)$ & $\mathrm{C} 63-\mathrm{H} 63 \mathrm{~A}$ & 0.9300 \\
\hline $\mathrm{C} 6-\mathrm{H} 6$ & 0.9300 & $\mathrm{C} 64-\mathrm{C} 65$ & $1.372(7)$ \\
\hline $\mathrm{C} 7-\mathrm{C} 11$ & $1.396(6)$ & $\mathrm{C} 65-\mathrm{C} 66$ & $1.375(7)$ \\
\hline $\mathrm{C} 7-\mathrm{C} 8$ & $1.402(7)$ & C65-H65 & 0.9300 \\
\hline $\mathrm{C} 8-\mathrm{C} 9$ & $1.359(8)$ & C66-H66 & 0.9300 \\
\hline $\mathrm{C} 8-\mathrm{H} 8$ & 0.9300 & $\mathrm{O} 73-\mathrm{C} 74$ & $1.363(11)$ \\
\hline $\mathrm{C} 9-\mathrm{C} 10$ & $1.397(8)$ & $\mathrm{O} 73-\mathrm{H} 73$ & 0.8200 \\
\hline C9-H9 & 0.9300 & O71-C77 & $1.294(12)$ \\
\hline $\mathrm{C} 10-\mathrm{H} 10$ & 0.9300 & $\mathrm{O} 72-\mathrm{C} 77$ & $1.321(12)$ \\
\hline $\mathrm{C} 11-\mathrm{C} 12$ & $1.439(6)$ & $\mathrm{C} 71-\mathrm{C} 72$ & $1.339(11)$ \\
\hline $\mathrm{C} 13-\mathrm{C} 14$ & $1.396(7)$ & $\mathrm{C} 71-\mathrm{C} 76$ & $1.410(12)$ \\
\hline $\mathrm{C} 13-\mathrm{H} 13$ & 0.9300 & C71-C77 & $1.487(13)$ \\
\hline $\mathrm{C} 14-\mathrm{C} 15$ & $1.352(8)$ & $\mathrm{C} 72-\mathrm{C} 73$ & $1.412(13)$ \\
\hline $\mathrm{C} 14-\mathrm{H} 14$ & 0.9300 & $\mathrm{C} 72-\mathrm{H} 72$ & 0.9300 \\
\hline $\mathrm{C} 15-\mathrm{C} 16$ & $1.382(8)$ & $\mathrm{C} 73-\mathrm{C} 74$ & $1.397(12)$ \\
\hline $\mathrm{C} 15-\mathrm{H} 15$ & 0.9300 & C $73-\mathrm{H} 71$ & 0.9300 \\
\hline $\mathrm{C} 16-\mathrm{C} 24$ & $1.417(6)$ & $\mathrm{C} 74-\mathrm{C} 75$ & $1.403(13)$ \\
\hline $\mathrm{C} 16-\mathrm{C} 17$ & $1.445(8)$ & $\mathrm{C} 75-\mathrm{C} 76$ & $1.454(13)$ \\
\hline $\mathrm{C} 17-\mathrm{C} 18$ & $1.341(9)$ & C75-H75 & 0.9300 \\
\hline $\mathrm{C} 17-\mathrm{H} 17$ & 0.9300 & $\mathrm{C} 76-\mathrm{H} 76$ & 0.9300 \\
\hline
\end{tabular}




\begin{tabular}{|c|c|c|c|}
\hline $\mathrm{C} 18-\mathrm{C} 19$ & $1.432(8)$ & $\mathrm{O} 1 \mathrm{~W}-\mathrm{H} 1 \mathrm{~A}$ & 0.97 \\
\hline C18-H18 & 0.9300 & $\mathrm{O} 1 \mathrm{~W}-\mathrm{H} 1 \mathrm{~B}$ & 1.00 \\
\hline $\mathrm{C} 19-\mathrm{C} 23$ & $1.404(7)$ & $\mathrm{O} 2 \mathrm{~W}-\mathrm{H} 2 \mathrm{~A}$ & 0.97 \\
\hline $\mathrm{C} 19-\mathrm{C} 20$ & $1.410(8)$ & $\mathrm{O} 2 \mathrm{~W}-\mathrm{H} 2 \mathrm{~B}$ & 0.83 \\
\hline $\mathrm{C} 20-\mathrm{C} 21$ & $1.342(8)$ & $\mathrm{O} 3 \mathrm{~W}-\mathrm{H} 3 \mathrm{~A}$ & 1.00 \\
\hline $\mathrm{C} 20-\mathrm{H} 20$ & 0.9300 & $\mathrm{O} 3 \mathrm{~W}-\mathrm{H} 3 \mathrm{~B}$ & 1.00 \\
\hline $\mathrm{C} 21-\mathrm{C} 22$ & $1.388(7)$ & $\mathrm{O} 4 \mathrm{~W}-\mathrm{H} 4 \mathrm{~A}$ & 1.01 \\
\hline $\mathrm{C} 21-\mathrm{H} 21$ & 0.9300 & $\mathrm{O} 4 \mathrm{~W}-\mathrm{H} 4 \mathrm{~B}$ & 0.86 \\
\hline $\mathrm{C} 22-\mathrm{H} 22$ & 0.9300 & $\mathrm{O} 5 \mathrm{~W}-\mathrm{H} 5 \mathrm{~A}$ & 0.96 \\
\hline $\mathrm{C} 23-\mathrm{C} 24$ & $1.437(7)$ & $\mathrm{O} 5 \mathrm{~W}-\mathrm{H} 5 \mathrm{~B}$ & 0.97 \\
\hline $\mathrm{N} 1-\mathrm{Cu} 1-\mathrm{N} 3$ & $176.67(15)$ & $\mathrm{N} 4-\mathrm{C} 22-\mathrm{C} 21$ & $123.3(5)$ \\
\hline $\mathrm{O} 53-\mathrm{Cu} 1-\mathrm{N} 2$ & $155.19(14)$ & $\mathrm{N} 4-\mathrm{C} 22-\mathrm{H} 22$ & 118.3 \\
\hline $\mathrm{O} 53-\mathrm{Cu} 1-\mathrm{N} 4$ & $101.39(14)$ & $\mathrm{C} 21-\mathrm{C} 22-\mathrm{H} 22$ & 118.3 \\
\hline $\mathrm{N} 2-\mathrm{Cu} 1-\mathrm{N} 4$ & $103.05(15)$ & $\mathrm{N} 4-\mathrm{C} 23-\mathrm{C} 19$ & $123.0(4)$ \\
\hline $\mathrm{O} 53-\mathrm{Cu} 1-\mathrm{N} 1$ & $89.92(14)$ & $\mathrm{N} 4-\mathrm{C} 23-\mathrm{C} 24$ & $116.7(4)$ \\
\hline $\mathrm{O} 53-\mathrm{Cu} 1-\mathrm{N} 3$ & $93.37(14)$ & $\mathrm{C} 19-\mathrm{C} 23-\mathrm{C} 24$ & $120.3(4)$ \\
\hline $\mathrm{N} 1-\mathrm{Cu} 1-\mathrm{N} 2$ & $81.74(14)$ & $\mathrm{N} 3-\mathrm{C} 24-\mathrm{C} 16$ & $122.0(4)$ \\
\hline $\mathrm{N} 3-\mathrm{Cu} 1-\mathrm{N} 2$ & $95.05(15)$ & $\mathrm{N} 3-\mathrm{C} 24-\mathrm{C} 23$ & $118.1(4)$ \\
\hline $\mathrm{N} 1-\mathrm{Cu} 1-\mathrm{N} 4$ & $100.08(14)$ & $\mathrm{C} 16-\mathrm{C} 24-\mathrm{C} 23$ & $119.9(4)$ \\
\hline $\mathrm{N} 3-\mathrm{Cu} 1-\mathrm{N} 4$ & $79.74(15)$ & $\mathrm{N} 5-\mathrm{C} 25-\mathrm{C} 26$ & $122.8(5)$ \\
\hline $\mathrm{N} 5-\mathrm{Cu} 2-\mathrm{N} 7$ & $173.07(16)$ & $\mathrm{N} 5-\mathrm{C} 25-\mathrm{H} 25$ & 118.6 \\
\hline $\mathrm{O} 51-\mathrm{Cu} 2-\mathrm{N} 5$ & $96.10(15)$ & $\mathrm{C} 26-\mathrm{C} 25-\mathrm{H} 25$ & 118.6 \\
\hline $\mathrm{O} 51-\mathrm{Cu} 2-\mathrm{N} 7$ & $89.77(15)$ & $\mathrm{C} 27-\mathrm{C} 26-\mathrm{C} 25$ & $119.5(6)$ \\
\hline $\mathrm{O} 51-\mathrm{Cu} 2-\mathrm{N} 8$ & $152.80(15)$ & $\mathrm{C} 27-\mathrm{C} 26-\mathrm{H} 26$ & 120.3 \\
\hline $\mathrm{N} 5-\mathrm{Cu} 2-\mathrm{N} 8$ & $94.96(16)$ & $\mathrm{C} 25-\mathrm{C} 26-\mathrm{H} 26$ & 120.3 \\
\hline $\mathrm{N} 7-\mathrm{Cu} 2-\mathrm{N} 8$ & $81.32(17)$ & $\mathrm{C} 26-\mathrm{C} 27-\mathrm{C} 28$ & $119.7(5)$ \\
\hline $\mathrm{O} 51-\mathrm{Cu} 2-\mathrm{N} 6$ & $101.29(16)$ & $\mathrm{C} 26-\mathrm{C} 27-\mathrm{H} 27$ & 120.1 \\
\hline $\mathrm{N} 5-\mathrm{Cu} 2-\mathrm{N} 6$ & $79.62(16)$ & $\mathrm{C} 28-\mathrm{C} 27-\mathrm{H} 27$ & 120.1 \\
\hline $\mathrm{N} 7-\mathrm{Cu} 2-\mathrm{N} 6$ & $95.64(16)$ & $\mathrm{C} 27-\mathrm{C} 28-\mathrm{C} 36$ & $117.9(5)$ \\
\hline $\mathrm{N} 8-\mathrm{Cu} 2-\mathrm{N} 6$ & $105.12(16)$ & $\mathrm{C} 27-\mathrm{C} 28-\mathrm{C} 29$ & $123.7(5)$ \\
\hline $\mathrm{C} 57-\mathrm{O} 51-\mathrm{Cu} 2$ & $112.6(3)$ & $\mathrm{C} 36-\mathrm{C} 28-\mathrm{C} 29$ & $118.5(6)$ \\
\hline $\mathrm{C} 54-\mathrm{O} 53-\mathrm{Cu} 1$ & $122.0(3)$ & $\mathrm{C} 30-\mathrm{C} 29-\mathrm{C} 28$ & $121.1(6)$ \\
\hline $\mathrm{C} 52-\mathrm{C} 51-\mathrm{C} 56$ & $117.5(4)$ & $\mathrm{C} 30-\mathrm{C} 29-\mathrm{H} 29$ & 119.5 \\
\hline $\mathrm{C} 52-\mathrm{C} 51-\mathrm{C} 57$ & $120.7(4)$ & $\mathrm{C} 28-\mathrm{C} 29-\mathrm{H} 29$ & 119.5 \\
\hline $\mathrm{C} 56-\mathrm{C} 51-\mathrm{C} 57$ & $121.7(4)$ & $\mathrm{C} 29-\mathrm{C} 30-\mathrm{C} 31$ & $121.6(5)$ \\
\hline $\mathrm{C} 53-\mathrm{C} 52-\mathrm{C} 51$ & $122.2(4)$ & $\mathrm{C} 29-\mathrm{C} 30-\mathrm{H} 30$ & 119.2 \\
\hline $\mathrm{C} 53-\mathrm{C} 52-\mathrm{H} 52$ & 118.9 & $\mathrm{C} 31-\mathrm{C} 30-\mathrm{H} 30$ & 119.2 \\
\hline $\mathrm{C} 51-\mathrm{C} 52-\mathrm{H} 52$ & 118.9 & $\mathrm{C} 35-\mathrm{C} 31-\mathrm{C} 32$ & $117.0(6)$ \\
\hline $\mathrm{C} 52-\mathrm{C} 53-\mathrm{C} 54$ & $121.0(4)$ & $\mathrm{C} 35-\mathrm{C} 31-\mathrm{C} 30$ & $118.9(6)$ \\
\hline $\mathrm{C} 52-\mathrm{C} 53-\mathrm{H} 53$ & 119.5 & $\mathrm{C} 32-\mathrm{C} 31-\mathrm{C} 30$ & $124.1(6)$ \\
\hline $\mathrm{C} 54-\mathrm{C} 53-\mathrm{H} 53$ & 119.5 & $\mathrm{C} 33-\mathrm{C} 32-\mathrm{C} 31$ & $120.0(6)$ \\
\hline $\mathrm{O} 53-\mathrm{C} 54-\mathrm{C} 53$ & $124.2(4)$ & $\mathrm{C} 33-\mathrm{C} 32-\mathrm{H} 32$ & 120.0 \\
\hline $\mathrm{O} 53-\mathrm{C} 54-\mathrm{C} 55$ & $118.6(4)$ & $\mathrm{C} 31-\mathrm{C} 32-\mathrm{H} 32$ & 120.0 \\
\hline $\mathrm{C} 53-\mathrm{C} 54-\mathrm{C} 55$ & $117.2(4)$ & $\mathrm{C} 32-\mathrm{C} 33-\mathrm{C} 34$ & $118.6(6)$ \\
\hline $\mathrm{C} 56-\mathrm{C} 55-\mathrm{C} 54$ & $120.2(4)$ & $\mathrm{C} 32-\mathrm{C} 33-\mathrm{H} 33$ & 120.7 \\
\hline $\mathrm{C} 56-\mathrm{C} 55-\mathrm{H} 55$ & 119.9 & $\mathrm{C} 34-\mathrm{C} 33-\mathrm{H} 33$ & 120.7 \\
\hline $\mathrm{C} 54-\mathrm{C} 55-\mathrm{H} 55$ & 119.9 & $\mathrm{~N} 6-\mathrm{C} 34-\mathrm{C} 33$ & $124.1(6)$ \\
\hline
\end{tabular}




\begin{tabular}{|c|c|}
\hline $\mathrm{C} 51-\mathrm{C} 56-\mathrm{C} 55$ & $121.7(5)$ \\
\hline $\mathrm{C} 51-\mathrm{C} 56-\mathrm{H} 56$ & 119.1 \\
\hline $\mathrm{C} 55-\mathrm{C} 56-\mathrm{H} 56$ & 119.1 \\
\hline $\mathrm{O} 52-\mathrm{C} 57-\mathrm{O} 51$ & $124.3(5)$ \\
\hline $\mathrm{O} 52-\mathrm{C} 57-\mathrm{C} 51$ & $119.7(5)$ \\
\hline $\mathrm{O} 51-\mathrm{C} 57-\mathrm{C} 51$ & $115.9(4)$ \\
\hline $\mathrm{C} 1-\mathrm{N} 1-\mathrm{C} 12$ & $118.2(4)$ \\
\hline $\mathrm{C} 1-\mathrm{N} 1-\mathrm{Cu} 1$ & $128.2(3)$ \\
\hline $\mathrm{C} 12-\mathrm{N} 1-\mathrm{Cu} 1$ & $113.5(3)$ \\
\hline $\mathrm{C} 10-\mathrm{N} 2-\mathrm{C} 11$ & 117.5 \\
\hline $\mathrm{C} 10-\mathrm{N} 2-\mathrm{Cu} 1$ & $130.8(3)$ \\
\hline $\mathrm{C} 11-\mathrm{N} 2-\mathrm{Cu} 1$ & $111.7(3)$ \\
\hline $\mathrm{C} 13-\mathrm{N} 3-\mathrm{C} 24$ & $118.5(4)$ \\
\hline $\mathrm{C} 13-\mathrm{N} 3-\mathrm{Cu} 1$ & $127.0(3)$ \\
\hline $\mathrm{C} 24-\mathrm{N} 3-\mathrm{Cu} 1$ & $114.4(3)$ \\
\hline $\mathrm{C} 22-\mathrm{N} 4-\mathrm{C} 23$ & $117.1(4)$ \\
\hline $\mathrm{C} 22-\mathrm{N} 4-\mathrm{Cu} 1$ & $132.1(3)$ \\
\hline $\mathrm{C} 23-\mathrm{N} 4-\mathrm{Cu} 1$ & $110.6(3)$ \\
\hline $\mathrm{C} 25-\mathrm{N} 5-\mathrm{C} 36$ & $117.7(4)$ \\
\hline $\mathrm{C} 25-\mathrm{N} 5-\mathrm{Cu} 2$ & $126.7(3)$ \\
\hline $\mathrm{C} 36-\mathrm{N} 5-\mathrm{Cu} 2$ & $115.6(3)$ \\
\hline $\mathrm{C} 34-\mathrm{N} 6-\mathrm{C} 35$ & $117.4(5)$ \\
\hline $\mathrm{C} 34-\mathrm{N} 6-\mathrm{Cu} 2$ & $132.7(4)$ \\
\hline $\mathrm{C} 35-\mathrm{N} 6-\mathrm{Cu} 2$ & $109.8(3)$ \\
\hline $\mathrm{C} 37-\mathrm{N} 7-\mathrm{C} 48$ & $118.3(4)$ \\
\hline $\mathrm{C} 37-\mathrm{N} 7-\mathrm{Cu} 2$ & $128.3(4)$ \\
\hline $\mathrm{C} 48-\mathrm{N} 7-\mathrm{Cu} 2$ & $113.4(3)$ \\
\hline $\mathrm{C} 46-\mathrm{N} 8-\mathrm{C} 47$ & 117.5 \\
\hline $\mathrm{C} 46-\mathrm{N} 8-\mathrm{Cu} 2$ & $131.0(4)$ \\
\hline $\mathrm{C} 47-\mathrm{N} 8-\mathrm{Cu} 2$ & $111.5(3)$ \\
\hline $\mathrm{N} 1-\mathrm{C} 1-\mathrm{C} 2$ & $122.1(4)$ \\
\hline $\mathrm{N} 1-\mathrm{C} 1-\mathrm{H} 1$ & 119.0 \\
\hline $\mathrm{C} 2-\mathrm{C} 1-\mathrm{H} 1$ & 119.0 \\
\hline $\mathrm{C} 3-\mathrm{C} 2-\mathrm{C} 1$ & $119.8(5)$ \\
\hline $\mathrm{C} 3-\mathrm{C} 2-\mathrm{H} 2$ & 120.1 \\
\hline $\mathrm{C} 1-\mathrm{C} 2-\mathrm{H} 2$ & 120.1 \\
\hline $\mathrm{C} 2-\mathrm{C} 3-\mathrm{C} 4$ & $120.1(4)$ \\
\hline $\mathrm{C} 2-\mathrm{C} 3-\mathrm{H} 3$ & 119.9 \\
\hline $\mathrm{C} 4-\mathrm{C} 3-\mathrm{H} 3$ & 119.9 \\
\hline $\mathrm{C} 12-\mathrm{C} 4-\mathrm{C} 3$ & $116.5(4)$ \\
\hline $\mathrm{C} 12-\mathrm{C} 4-\mathrm{C} 5$ & $118.3(5)$ \\
\hline $\mathrm{C} 3-\mathrm{C} 4-\mathrm{C} 5$ & $125.2(4)$ \\
\hline $\mathrm{C} 6-\mathrm{C} 5-\mathrm{C} 4$ & $121.7(5)$ \\
\hline $\mathrm{C} 6-\mathrm{C} 5-\mathrm{H} 5$ & 119.1 \\
\hline $\mathrm{C} 4-\mathrm{C} 5-\mathrm{H} 5$ & 119.1 \\
\hline $\mathrm{C} 5-\mathrm{C} 6-\mathrm{C} 7$ & $121.2(5)$ \\
\hline $\mathrm{C} 5-\mathrm{C} 6-\mathrm{H} 6$ & 119.4 \\
\hline $\mathrm{C} 7-\mathrm{C} 6-\mathrm{H} 6$ & 119.4 \\
\hline
\end{tabular}

\begin{tabular}{|c|c|}
\hline $\mathrm{N} 6-\mathrm{C} 34-\mathrm{H} 34$ & 117.9 \\
\hline $\mathrm{C} 33-\mathrm{C} 34-\mathrm{H} 34$ & 117.9 \\
\hline $\mathrm{N} 6-\mathrm{C} 35-\mathrm{C} 31$ & $122.8(5)$ \\
\hline $\mathrm{N} 6-\mathrm{C} 35-\mathrm{C} 36$ & $117.3(4)$ \\
\hline $\mathrm{C} 31-\mathrm{C} 35-\mathrm{C} 36$ & $119.8(5)$ \\
\hline $\mathrm{N} 5-\mathrm{C} 36-\mathrm{C} 28$ & $122.4(5)$ \\
\hline $\mathrm{N} 5-\mathrm{C} 36-\mathrm{C} 35$ & $117.5(4)$ \\
\hline $\mathrm{C} 28-\mathrm{C} 36-\mathrm{C} 35$ & $120.1(5)$ \\
\hline N7-C37-C38 & $122.2(5)$ \\
\hline N7-C37-H37 & 118.9 \\
\hline $\mathrm{C} 38-\mathrm{C} 37-\mathrm{H} 37$ & 118.9 \\
\hline $\mathrm{C} 39-\mathrm{C} 38-\mathrm{C} 37$ & $120.5(6)$ \\
\hline $\mathrm{C} 39-\mathrm{C} 38-\mathrm{H} 38$ & 119.8 \\
\hline $\mathrm{C} 37-\mathrm{C} 38-\mathrm{H} 38$ & 119.8 \\
\hline $\mathrm{C} 38-\mathrm{C} 39-\mathrm{C} 40$ & $119.6(5)$ \\
\hline $\mathrm{C} 38-\mathrm{C} 39-\mathrm{H} 39$ & 120.2 \\
\hline $\mathrm{C} 40-\mathrm{C} 39-\mathrm{H} 39$ & 120.2 \\
\hline $\mathrm{C} 48-\mathrm{C} 40-\mathrm{C} 39$ & $116.6(5)$ \\
\hline $\mathrm{C} 48-\mathrm{C} 40-\mathrm{C} 41$ & $118.5(5)$ \\
\hline $\mathrm{C} 39-\mathrm{C} 40-\mathrm{C} 41$ & $124.9(5)$ \\
\hline $\mathrm{C} 42-\mathrm{C} 41-\mathrm{C} 40$ & $121.2(5)$ \\
\hline $\mathrm{C} 42-\mathrm{C} 41-\mathrm{H} 41$ & 119.4 \\
\hline $\mathrm{C} 40-\mathrm{C} 41-\mathrm{H} 41$ & 119.4 \\
\hline $\mathrm{C} 41-\mathrm{C} 42-\mathrm{C} 43$ & $122.4(5)$ \\
\hline $\mathrm{C} 41-\mathrm{C} 42-\mathrm{H} 42$ & 118.8 \\
\hline $\mathrm{C} 43-\mathrm{C} 42-\mathrm{H} 42$ & 118.8 \\
\hline $\mathrm{C} 44-\mathrm{C} 43-\mathrm{C} 47$ & $117.3(5)$ \\
\hline $\mathrm{C} 44-\mathrm{C} 43-\mathrm{C} 42$ & $125.5(5)$ \\
\hline $\mathrm{C} 47-\mathrm{C} 43-\mathrm{C} 42$ & $117.2(5)$ \\
\hline $\mathrm{C} 45-\mathrm{C} 44-\mathrm{C} 43$ & $120.2(5)$ \\
\hline $\mathrm{C} 45-\mathrm{C} 44-\mathrm{H} 44$ & 119.9 \\
\hline $\mathrm{C} 43-\mathrm{C} 44-\mathrm{H} 44$ & 119.9 \\
\hline $\mathrm{C} 44-\mathrm{C} 45-\mathrm{C} 46$ & $118.8(6)$ \\
\hline $\mathrm{C} 44-\mathrm{C} 45-\mathrm{H} 45$ & 120.6 \\
\hline $\mathrm{C} 46-\mathrm{C} 45-\mathrm{H} 45$ & 120.6 \\
\hline $\mathrm{N} 8-\mathrm{C} 46-\mathrm{C} 45$ & $123.4(6)$ \\
\hline $\mathrm{N} 8-\mathrm{C} 46-\mathrm{H} 46$ & 118.3 \\
\hline $\mathrm{C} 45-\mathrm{C} 46-\mathrm{H} 46$ & 118.3 \\
\hline $\mathrm{N} 8-\mathrm{C} 47-\mathrm{C} 43$ & $122.8(5)$ \\
\hline $\mathrm{N} 8-\mathrm{C} 47-\mathrm{C} 48$ & $116.9(4)$ \\
\hline $\mathrm{C} 43-\mathrm{C} 47-\mathrm{C} 48$ & $120.3(5)$ \\
\hline $\mathrm{N} 7-\mathrm{C} 48-\mathrm{C} 40$ & $122.8(5)$ \\
\hline $\mathrm{N} 7-\mathrm{C} 48-\mathrm{C} 47$ & $116.8(4)$ \\
\hline $\mathrm{C} 40-\mathrm{C} 48-\mathrm{C} 47$ & $120.3(5)$ \\
\hline $\mathrm{C} 64-\mathrm{O} 63-\mathrm{H} 63$ & 109.5 \\
\hline $\mathrm{C} 66-\mathrm{C} 61-\mathrm{C} 62$ & $117.5(4)$ \\
\hline C66-C61-C67 & $120.5(5)$ \\
\hline C62-C61-C67 & $122.0(4)$ \\
\hline
\end{tabular}




\begin{tabular}{|c|c|c|c|}
\hline $\mathrm{C} 11-\mathrm{C} 7-\mathrm{C} 8$ & $117.5(5)$ & $\mathrm{C} 63-\mathrm{C} 62-\mathrm{C} 61$ & $122.0(5)$ \\
\hline $\mathrm{C} 11-\mathrm{C} 7-\mathrm{C} 6$ & $118.3(5)$ & $\mathrm{C} 63-\mathrm{C} 62-\mathrm{H} 62$ & 119.0 \\
\hline $\mathrm{C} 8-\mathrm{C} 7-\mathrm{C} 6$ & $124.2(5)$ & $\mathrm{C} 61-\mathrm{C} 62-\mathrm{H} 62$ & 119.0 \\
\hline $\mathrm{C} 9-\mathrm{C} 8-\mathrm{C} 7$ & $118.8(5)$ & $\mathrm{C} 62-\mathrm{C} 63-\mathrm{C} 64$ & $119.5(5)$ \\
\hline $\mathrm{C} 9-\mathrm{C} 8-\mathrm{H} 8$ & 120.6 & $\mathrm{C} 62-\mathrm{C} 63-\mathrm{H} 63 \mathrm{~A}$ & 120.3 \\
\hline $\mathrm{C} 7-\mathrm{C} 8-\mathrm{H} 8$ & 120.6 & C64-C63-H63A & 120.3 \\
\hline $\mathrm{C} 8-\mathrm{C} 9-\mathrm{C} 10$ & $120.4(5)$ & $\mathrm{O} 63-\mathrm{C} 64-\mathrm{C} 65$ & $118.8(5)$ \\
\hline $\mathrm{C} 8-\mathrm{C} 9-\mathrm{H} 9$ & 119.8 & $\mathrm{O} 63-\mathrm{C} 64-\mathrm{C} 63$ & $122.0(5)$ \\
\hline $\mathrm{C} 10-\mathrm{C} 9-\mathrm{H} 9$ & 119.8 & $\mathrm{C} 65-\mathrm{C} 64-\mathrm{C} 63$ & $119.1(5)$ \\
\hline $\mathrm{N} 2-\mathrm{C} 10-\mathrm{C} 9$ & $122.4(5)$ & C64-C65-C66 & $121.0(5)$ \\
\hline $\mathrm{N} 2-\mathrm{C} 10-\mathrm{H} 10$ & 118.8 & $\mathrm{C} 64-\mathrm{C} 65-\mathrm{H} 65$ & 119.5 \\
\hline $\mathrm{C} 9-\mathrm{C} 10-\mathrm{H} 10$ & 118.8 & $\mathrm{C} 66-\mathrm{C} 65-\mathrm{H} 65$ & 119.5 \\
\hline $\mathrm{N} 2-\mathrm{C} 11-\mathrm{C} 7$ & $123.4(4)$ & C65-C66-C61 & $120.9(5)$ \\
\hline $\mathrm{N} 2-\mathrm{C} 11-\mathrm{C} 12$ & $116.4(4)$ & C65-C66-H66 & 119.6 \\
\hline $\mathrm{C} 7-\mathrm{C} 11-\mathrm{C} 12$ & $120.2(4)$ & C61-C66-H66 & 119.6 \\
\hline $\mathrm{N} 1-\mathrm{C} 12-\mathrm{C} 4$ & $123.3(4)$ & $\mathrm{O} 61-\mathrm{C} 67-\mathrm{O} 62$ & $124.3(5)$ \\
\hline $\mathrm{N} 1-\mathrm{C} 12-\mathrm{C} 11$ & $116.6(4)$ & $\mathrm{O} 61-\mathrm{C} 67-\mathrm{C} 61$ & $119.5(4)$ \\
\hline $\mathrm{C} 4-\mathrm{C} 12-\mathrm{C} 11$ & $120.1(4)$ & $\mathrm{O} 62-\mathrm{C} 67-\mathrm{C} 61$ & $116.2(5)$ \\
\hline $\mathrm{N} 3-\mathrm{C} 13-\mathrm{C} 14$ & $122.5(5)$ & $\mathrm{C} 74-\mathrm{O} 73-\mathrm{H} 73$ & 109.5 \\
\hline $\mathrm{N} 3-\mathrm{C} 13-\mathrm{H} 13$ & 118.8 & $\mathrm{C} 72-\mathrm{C} 71-\mathrm{C} 76$ & $120.4(10)$ \\
\hline $\mathrm{C} 14-\mathrm{C} 13-\mathrm{H} 13$ & 118.8 & $\mathrm{C} 72-\mathrm{C} 71-\mathrm{C} 77$ & $120.5(11)$ \\
\hline $\mathrm{C} 15-\mathrm{C} 14-\mathrm{C} 13$ & $119.2(5)$ & $\mathrm{C} 76-\mathrm{C} 71-\mathrm{C} 77$ & $119.0(9)$ \\
\hline $\mathrm{C} 15-\mathrm{C} 14-\mathrm{H} 14$ & 120.4 & $\mathrm{C} 71-\mathrm{C} 72-\mathrm{C} 73$ & $121.9(10)$ \\
\hline $\mathrm{C} 13-\mathrm{C} 14-\mathrm{H} 14$ & 120.4 & $\mathrm{C} 71-\mathrm{C} 72-\mathrm{H} 72$ & 119.0 \\
\hline $\mathrm{C} 14-\mathrm{C} 15-\mathrm{C} 16$ & $120.7(5)$ & $\mathrm{C} 73-\mathrm{C} 72-\mathrm{H} 72$ & 119.0 \\
\hline $\mathrm{C} 14-\mathrm{C} 15-\mathrm{H} 15$ & 119.7 & $\mathrm{C} 74-\mathrm{C} 73-\mathrm{C} 72$ & $120.2(9)$ \\
\hline $\mathrm{C} 16-\mathrm{C} 15-\mathrm{H} 15$ & 119.7 & $\mathrm{C} 74-\mathrm{C} 73-\mathrm{H} 71$ & 119.9 \\
\hline $\mathrm{C} 15-\mathrm{C} 16-\mathrm{C} 24$ & $117.1(5)$ & $\mathrm{C} 72-\mathrm{C} 73-\mathrm{H} 71$ & 119.9 \\
\hline $\mathrm{C} 15-\mathrm{C} 16-\mathrm{C} 17$ & $125.4(5)$ & $\mathrm{O} 73-\mathrm{C} 74-\mathrm{C} 73$ & $124.4(10)$ \\
\hline $\mathrm{C} 24-\mathrm{C} 16-\mathrm{C} 17$ & $117.5(5)$ & $\mathrm{O} 73-\mathrm{C} 74-\mathrm{C} 75$ & $116.3(10)$ \\
\hline $\mathrm{C} 18-\mathrm{C} 17-\mathrm{C} 16$ & $122.4(5)$ & $\mathrm{C} 73-\mathrm{C} 74-\mathrm{C} 75$ & $119.1(10)$ \\
\hline $\mathrm{C} 18-\mathrm{C} 17-\mathrm{H} 17$ & 118.8 & $\mathrm{C} 74-\mathrm{C} 75-\mathrm{C} 76$ & $119.5(9)$ \\
\hline $\mathrm{C} 16-\mathrm{C} 17-\mathrm{H} 17$ & 118.8 & $\mathrm{C} 74-\mathrm{C} 75-\mathrm{H} 75$ & 120.2 \\
\hline $\mathrm{C} 17-\mathrm{C} 18-\mathrm{C} 19$ & $121.0(5)$ & $\mathrm{C} 76-\mathrm{C} 75-\mathrm{H} 75$ & 120.2 \\
\hline $\mathrm{C} 17-\mathrm{C} 18-\mathrm{H} 18$ & 119.5 & $\mathrm{C} 71-\mathrm{C} 76-\mathrm{C} 75$ & $118.7(10)$ \\
\hline $\mathrm{C} 19-\mathrm{C} 18-\mathrm{H} 18$ & 119.5 & $\mathrm{C} 71-\mathrm{C} 76-\mathrm{H} 76$ & 120.7 \\
\hline $\mathrm{C} 23-\mathrm{C} 19-\mathrm{C} 20$ & $117.0(5)$ & $\mathrm{C} 75-\mathrm{C} 76-\mathrm{H} 76$ & 120.7 \\
\hline $\mathrm{C} 23-\mathrm{C} 19-\mathrm{C} 18$ & $118.8(5)$ & $\mathrm{O} 71-\mathrm{C} 77-\mathrm{O} 72$ & $125.2(9)$ \\
\hline $\mathrm{C} 20-\mathrm{C} 19-\mathrm{C} 18$ & $124.1(5)$ & $\mathrm{O} 71-\mathrm{C} 77-\mathrm{C} 71$ & $115.3(10)$ \\
\hline $\mathrm{C} 21-\mathrm{C} 20-\mathrm{C} 19$ & $119.3(5)$ & $\mathrm{O} 72-\mathrm{C} 77-\mathrm{C} 71$ & $119.4(10)$ \\
\hline $\mathrm{C} 21-\mathrm{C} 20-\mathrm{H} 20$ & 120.3 & $\mathrm{H} 1 \mathrm{~A}-\mathrm{O} 1 \mathrm{~W}-\mathrm{H} 1 \mathrm{~B}$ & 103 \\
\hline $\mathrm{C} 19-\mathrm{C} 20-\mathrm{H} 20$ & 120.3 & $\mathrm{H} 2 \mathrm{~A}-\mathrm{O} 2 \mathrm{~W}-\mathrm{H} 2 \mathrm{~B}$ & 121 \\
\hline $\mathrm{C} 20-\mathrm{C} 21-\mathrm{C} 22$ & $120.1(5)$ & $\mathrm{H} 3 \mathrm{~A}-\mathrm{O} 3 \mathrm{~W}-\mathrm{H} 3 \mathrm{~B}$ & 102 \\
\hline $\mathrm{C} 20-\mathrm{C} 21-\mathrm{H} 21$ & 119.9 & $\mathrm{H} 4 \mathrm{~A}-\mathrm{O} 4 \mathrm{~W}-\mathrm{H} 4 \mathrm{~B}$ & 107 \\
\hline $\mathrm{C} 22-\mathrm{C} 21-\mathrm{H} 21$ & 119.9 & $\mathrm{H} 5 \mathrm{~A}-\mathrm{O} 5 \mathrm{~W}-\mathrm{H} 5 \mathrm{~B}$ & 114 \\
\hline $\mathrm{C} 56-\mathrm{C} 51-\mathrm{C} 52-\mathrm{C} 53$ & $0.5(7)$ & $\mathrm{C} 26-\mathrm{C} 27-\mathrm{C} 28-\mathrm{C} 29$ & $178.1(5)$ \\
\hline $\mathrm{C} 57-\mathrm{C} 51-\mathrm{C} 52-\mathrm{C} 53$ & $-174.7(4)$ & $\mathrm{C} 27-\mathrm{C} 28-\mathrm{C} 29-\mathrm{C} 30$ & $-177.7(5)$ \\
\hline
\end{tabular}




\begin{tabular}{|c|c|c|c|}
\hline $\mathrm{C} 51-\mathrm{C} 52-\mathrm{C} 53-\mathrm{C} 54$ & $3.8(7)$ & $\mathrm{C} 36-\mathrm{C} 28-\mathrm{C} 29-\mathrm{C} 30$ & $1.2(8)$ \\
\hline $\mathrm{Cu} 1-\mathrm{O} 53-\mathrm{C} 54-\mathrm{C} 53$ & $12.0(5)$ & $\mathrm{C} 28-\mathrm{C} 29-\mathrm{C} 30-\mathrm{C} 31$ & $-2.5(9)$ \\
\hline $\mathrm{Cu} 1-\mathrm{O} 53-\mathrm{C} 54-\mathrm{C} 55$ & $-170.4(3)$ & $\mathrm{C} 29-\mathrm{C} 30-\mathrm{C} 31-\mathrm{C} 35$ & $1.9(8)$ \\
\hline $\mathrm{C} 52-\mathrm{C} 53-\mathrm{C} 54-\mathrm{O} 53$ & $172.1(4)$ & $\mathrm{C} 29-\mathrm{C} 30-\mathrm{C} 31-\mathrm{C} 32$ & $-179.4(6)$ \\
\hline $\mathrm{C} 52-\mathrm{C} 53-\mathrm{C} 54-\mathrm{C} 55$ & $-5.6(6)$ & $\mathrm{C} 35-\mathrm{C} 31-\mathrm{C} 32-\mathrm{C} 33$ & $-0.9(9)$ \\
\hline $\mathrm{O} 53-\mathrm{C} 54-\mathrm{C} 55-\mathrm{C} 56$ & $-174.6(4)$ & $\mathrm{C} 30-\mathrm{C} 31-\mathrm{C} 32-\mathrm{C} 33$ & $-179.7(6)$ \\
\hline $\mathrm{C} 53-\mathrm{C} 54-\mathrm{C} 55-\mathrm{C} 56$ & $3.2(6)$ & $\mathrm{C} 31-\mathrm{C} 32-\mathrm{C} 33-\mathrm{C} 34$ & $-0.2(10)$ \\
\hline $\mathrm{C} 52-\mathrm{C} 51-\mathrm{C} 56-\mathrm{C} 55$ & $-2.9(7)$ & $\mathrm{C} 35-\mathrm{N} 6-\mathrm{C} 34-\mathrm{C} 33$ & $-0.3(9)$ \\
\hline $\mathrm{C} 57-\mathrm{C} 51-\mathrm{C} 56-\mathrm{C} 55$ & $172.2(4)$ & $\mathrm{Cu} 2-\mathrm{N} 6-\mathrm{C} 34-\mathrm{C} 33$ & $174.4(5)$ \\
\hline $\mathrm{C} 54-\mathrm{C} 55-\mathrm{C} 56-\mathrm{C} 51$ & $1.0(7)$ & $\mathrm{C} 32-\mathrm{C} 33-\mathrm{C} 34-\mathrm{N} 6$ & $0.9(10)$ \\
\hline $\mathrm{Cu} 2-\mathrm{O} 51-\mathrm{C} 57-\mathrm{O} 52$ & $6.4(6)$ & $\mathrm{C} 34-\mathrm{N} 6-\mathrm{C} 35-\mathrm{C} 31$ & $-0.9(7)$ \\
\hline $\mathrm{Cu} 2-\mathrm{O} 51-\mathrm{C} 57-\mathrm{C} 51$ & $-171.7(3)$ & $\mathrm{Cu} 2-\mathrm{N} 6-\mathrm{C} 35-\mathrm{C} 31$ & $-176.8(4)$ \\
\hline $\mathrm{C} 52-\mathrm{C} 51-\mathrm{C} 57-\mathrm{O} 52$ & $22.8(6)$ & $\mathrm{C} 34-\mathrm{N} 6-\mathrm{C} 35-\mathrm{C} 36$ & $179.5(5)$ \\
\hline $\mathrm{C} 56-\mathrm{C} 51-\mathrm{C} 57-\mathrm{O} 52$ & $-152.2(5)$ & $\mathrm{Cu} 2-\mathrm{N} 6-\mathrm{C} 35-\mathrm{C} 36$ & $3.6(5)$ \\
\hline $\mathrm{C} 52-\mathrm{C} 51-\mathrm{C} 57-\mathrm{O} 51$ & $-158.9(4)$ & $\mathrm{C} 32-\mathrm{C} 31-\mathrm{C} 35-\mathrm{N} 6$ & $1.6(7)$ \\
\hline $\mathrm{C} 56-\mathrm{C} 51-\mathrm{C} 57-\mathrm{O} 51$ & $26.1(6)$ & $\mathrm{C} 30-\mathrm{C} 31-\mathrm{C} 35-\mathrm{N} 6$ & $-179.6(5)$ \\
\hline $\mathrm{C} 12-\mathrm{N} 1-\mathrm{C} 1-\mathrm{C} 2$ & $0.8(7)$ & $\mathrm{C} 32-\mathrm{C} 31-\mathrm{C} 35-\mathrm{C} 36$ & $-178.8(5)$ \\
\hline $\mathrm{Cu} 1-\mathrm{N} 1-\mathrm{C} 1-\mathrm{C} 2$ & $179.3(3)$ & $\mathrm{C} 30-\mathrm{C} 31-\mathrm{C} 35-\mathrm{C} 36$ & $0.0(7)$ \\
\hline $\mathrm{N} 1-\mathrm{C} 1-\mathrm{C} 2-\mathrm{C} 3$ & $-0.2(7)$ & $\mathrm{C} 25-\mathrm{N} 5-\mathrm{C} 36-\mathrm{C} 28$ & $-0.4(7)$ \\
\hline $\mathrm{C} 1-\mathrm{C} 2-\mathrm{C} 3-\mathrm{C} 4$ & $-0.1(8)$ & $\mathrm{Cu} 2-\mathrm{N} 5-\mathrm{C} 36-\mathrm{C} 28$ & $179.0(4)$ \\
\hline $\mathrm{C} 2-\mathrm{C} 3-\mathrm{C} 4-\mathrm{C} 12$ & $-0.1(7)$ & $\mathrm{C} 25-\mathrm{N} 5-\mathrm{C} 36-\mathrm{C} 35$ & $-179.3(4)$ \\
\hline $\mathrm{C} 2-\mathrm{C} 3-\mathrm{C} 4-\mathrm{C} 5$ & $177.8(5)$ & $\mathrm{Cu} 2-\mathrm{N} 5-\mathrm{C} 36-\mathrm{C} 35$ & $0.2(5)$ \\
\hline $\mathrm{C} 12-\mathrm{C} 4-\mathrm{C} 5-\mathrm{C} 6$ & $0.3(8)$ & $\mathrm{C} 27-\mathrm{C} 28-\mathrm{C} 36-\mathrm{N} 5$ & $0.8(7)$ \\
\hline $\mathrm{C} 3-\mathrm{C} 4-\mathrm{C} 5-\mathrm{C} 6$ & $-177.6(5)$ & $\mathrm{C} 29-\mathrm{C} 28-\mathrm{C} 36-\mathrm{N} 5$ & $-178.2(4)$ \\
\hline $\mathrm{C} 4-\mathrm{C} 5-\mathrm{C} 6-\mathrm{C} 7$ & $-2.9(9)$ & $\mathrm{C} 27-\mathrm{C} 28-\mathrm{C} 36-\mathrm{C} 35$ & $179.7(4)$ \\
\hline $\mathrm{C} 5-\mathrm{C} 6-\mathrm{C} 7-\mathrm{C} 11$ & $3.4(8)$ & $\mathrm{C} 29-\mathrm{C} 28-\mathrm{C} 36-\mathrm{C} 35$ & $0.7(7)$ \\
\hline $\mathrm{C} 5-\mathrm{C} 6-\mathrm{C} 7-\mathrm{C} 8$ & $-175.8(5)$ & $\mathrm{N} 6-\mathrm{C} 35-\mathrm{C} 36-\mathrm{N} 5$ & $-2.7(6)$ \\
\hline $\mathrm{C} 11-\mathrm{C} 7-\mathrm{C} 8-\mathrm{C} 9$ & $0.1(8)$ & $\mathrm{C} 31-\mathrm{C} 35-\mathrm{C} 36-\mathrm{N} 5$ & $177.7(4)$ \\
\hline $\mathrm{C} 6-\mathrm{C} 7-\mathrm{C} 8-\mathrm{C} 9$ & $179.3(5)$ & $\mathrm{N} 6-\mathrm{C} 35-\mathrm{C} 36-\mathrm{C} 28$ & $178.4(4)$ \\
\hline $\mathrm{C} 7-\mathrm{C} 8-\mathrm{C} 9-\mathrm{C} 10$ & $0.7(9)$ & $\mathrm{C} 31-\mathrm{C} 35-\mathrm{C} 36-\mathrm{C} 28$ & $-1.2(7)$ \\
\hline $\mathrm{C} 11-\mathrm{N} 2-\mathrm{C} 10-\mathrm{C} 9$ & $-0.6(8)$ & $\mathrm{C} 48-\mathrm{N} 7-\mathrm{C} 37-\mathrm{C} 38$ & $0.0(8)$ \\
\hline $\mathrm{Cu} 1-\mathrm{N} 2-\mathrm{C} 10-\mathrm{C} 9$ & $178.5(4)$ & $\mathrm{Cu} 2-\mathrm{N} 7-\mathrm{C} 37-\mathrm{C} 38$ & $-179.3(4)$ \\
\hline $\mathrm{C} 8-\mathrm{C} 9-\mathrm{C} 10-\mathrm{N} 2$ & $-0.5(9)$ & $\mathrm{N} 7-\mathrm{C} 37-\mathrm{C} 38-\mathrm{C} 39$ & $0.7(9)$ \\
\hline $\mathrm{C} 10-\mathrm{N} 2-\mathrm{C} 11-\mathrm{C} 7$ & $1.5(7)$ & $\mathrm{C} 37-\mathrm{C} 38-\mathrm{C} 39-\mathrm{C} 40$ & $0.1(9)$ \\
\hline $\mathrm{Cu} 1-\mathrm{N} 2-\mathrm{C} 11-\mathrm{C} 7$ & $-177.8(4)$ & $\mathrm{C} 38-\mathrm{C} 39-\mathrm{C} 40-\mathrm{C} 48$ & $-1.5(8)$ \\
\hline $\mathrm{C} 10-\mathrm{N} 2-\mathrm{C} 11-\mathrm{C} 12$ & $-177.7(4)$ & $\mathrm{C} 38-\mathrm{C} 39-\mathrm{C} 40-\mathrm{C} 41$ & $177.8(5)$ \\
\hline $\mathrm{Cu} 1-\mathrm{N} 2-\mathrm{C} 11-\mathrm{C} 12$ & $3.0(5)$ & $\mathrm{C} 48-\mathrm{C} 40-\mathrm{C} 41-\mathrm{C} 42$ & $-3.6(8)$ \\
\hline $\mathrm{C} 8-\mathrm{C} 7-\mathrm{C} 11-\mathrm{N} 2$ & $-1.2(7)$ & $\mathrm{C} 39-\mathrm{C} 40-\mathrm{C} 41-\mathrm{C} 42$ & $177.2(6)$ \\
\hline $\mathrm{C} 6-\mathrm{C} 7-\mathrm{C} 11-\mathrm{N} 2$ & $179.5(4)$ & $\mathrm{C} 40-\mathrm{C} 41-\mathrm{C} 42-\mathrm{C} 43$ & $2.6(9)$ \\
\hline $\mathrm{C} 8-\mathrm{C} 7-\mathrm{C} 11-\mathrm{C} 12$ & $177.9(4)$ & $\mathrm{C} 41-\mathrm{C} 42-\mathrm{C} 43-\mathrm{C} 44$ & $178.2(6)$ \\
\hline $\mathrm{C} 6-\mathrm{C} 7-\mathrm{C} 11-\mathrm{C} 12$ & $-1.3(7)$ & $\mathrm{C} 41-\mathrm{C} 42-\mathrm{C} 43-\mathrm{C} 47$ & $-0.1(8)$ \\
\hline $\mathrm{C} 1-\mathrm{N} 1-\mathrm{C} 12-\mathrm{C} 4$ & $-1.0(7)$ & $\mathrm{C} 47-\mathrm{C} 43-\mathrm{C} 44-\mathrm{C} 45$ & $1.4(9)$ \\
\hline $\mathrm{Cu} 1-\mathrm{N} 1-\mathrm{C} 12-\mathrm{C} 4$ & $-179.8(3)$ & $\mathrm{C} 42-\mathrm{C} 43-\mathrm{C} 44-\mathrm{C} 45$ & $-176.9(6)$ \\
\hline $\mathrm{C} 1-\mathrm{N} 1-\mathrm{C} 12-\mathrm{C} 11$ & $179.8(4)$ & $\mathrm{C} 43-\mathrm{C} 44-\mathrm{C} 45-\mathrm{C} 46$ & $0.0(9)$ \\
\hline $\mathrm{Cu} 1-\mathrm{N} 1-\mathrm{C} 12-\mathrm{C} 11$ & $1.1(5)$ & $\mathrm{C} 47-\mathrm{N} 8-\mathrm{C} 46-\mathrm{C} 45$ & $-0.1(8)$ \\
\hline $\mathrm{C} 3-\mathrm{C} 4-\mathrm{C} 12-\mathrm{N} 1$ & $0.7(7)$ & $\mathrm{Cu} 2-\mathrm{N} 8-\mathrm{C} 46-\mathrm{C} 45$ & $-179.2(4)$ \\
\hline $\mathrm{C} 5-\mathrm{C} 4-\mathrm{C} 12-\mathrm{N} 1$ & $-177.4(4)$ & $\mathrm{C} 44-\mathrm{C} 45-\mathrm{C} 46-\mathrm{N} 8$ & $-0.7(9)$ \\
\hline $\mathrm{C} 3-\mathrm{C} 4-\mathrm{C} 12-\mathrm{C} 11$ & $179.9(4)$ & $\mathrm{C} 46-\mathrm{N} 8-\mathrm{C} 47-\mathrm{C} 43$ & $1.6(7)$ \\
\hline
\end{tabular}




\begin{tabular}{|c|c|}
\hline $\mathrm{C} 5-\mathrm{C} 4-\mathrm{C} 12-\mathrm{C} 11$ & $1.8(7)$ \\
\hline $\mathrm{N} 2-\mathrm{C} 11-\mathrm{C} 12-\mathrm{N} 1$ & $-2.8(6)$ \\
\hline $\mathrm{C} 7-\mathrm{C} 11-\mathrm{C} 12-\mathrm{N} 1$ & $178.0(4)$ \\
\hline $\mathrm{N} 2-\mathrm{C} 11-\mathrm{C} 12-\mathrm{C} 4$ & $178.0(4)$ \\
\hline $\mathrm{C} 7-\mathrm{C} 11-\mathrm{C} 12-\mathrm{C} 4$ & $-1.2(7)$ \\
\hline $\mathrm{C} 24-\mathrm{N} 3-\mathrm{C} 13-\mathrm{C} 14$ & $0.2(8)$ \\
\hline $\mathrm{Cu} 1-\mathrm{N} 3-\mathrm{C} 13-\mathrm{C} 14$ & $-175.7(4)$ \\
\hline $\mathrm{N} 3-\mathrm{C} 13-\mathrm{C} 14-\mathrm{C} 15$ & $0.2(8)$ \\
\hline $\mathrm{C} 13-\mathrm{C} 14-\mathrm{C} 15-\mathrm{C} 16$ & $0.9(9)$ \\
\hline $\mathrm{C} 14-\mathrm{C} 15-\mathrm{C} 16-\mathrm{C} 24$ & $-2.4(8)$ \\
\hline $\mathrm{C} 14-\mathrm{C} 15-\mathrm{C} 16-\mathrm{C} 17$ & $178.1(5)$ \\
\hline $\mathrm{C} 15-\mathrm{C} 16-\mathrm{C} 17-\mathrm{C} 18$ & $178.6(6)$ \\
\hline $\mathrm{C} 24-\mathrm{C} 16-\mathrm{C} 17-\mathrm{C} 18$ & $-0.9(8)$ \\
\hline $\mathrm{C} 16-\mathrm{C} 17-\mathrm{C} 18-\mathrm{C} 19$ & $-1.9(9)$ \\
\hline $\mathrm{C} 17-\mathrm{C} 18-\mathrm{C} 19-\mathrm{C} 23$ & $3.8(8)$ \\
\hline $\mathrm{C} 17-\mathrm{C} 18-\mathrm{C} 19-\mathrm{C} 20$ & $-176.9(6)$ \\
\hline $\mathrm{C} 23-\mathrm{C} 19-\mathrm{C} 20-\mathrm{C} 21$ & $0.7(8)$ \\
\hline $\mathrm{C} 18-\mathrm{C} 19-\mathrm{C} 20-\mathrm{C} 21$ & $-178.5(5)$ \\
\hline $\mathrm{C} 19-\mathrm{C} 20-\mathrm{C} 21-\mathrm{C} 22$ & $0.3(9)$ \\
\hline $\mathrm{C} 23-\mathrm{N} 4-\mathrm{C} 22-\mathrm{C} 21$ & $0.1(7)$ \\
\hline $\mathrm{Cu} 1-\mathrm{N} 4-\mathrm{C} 22-\mathrm{C} 21$ & $174.9(4)$ \\
\hline $\mathrm{C} 20-\mathrm{C} 21-\mathrm{C} 22-\mathrm{N} 4$ & $-0.7(9)$ \\
\hline $\mathrm{C} 22-\mathrm{N} 4-\mathrm{C} 23-\mathrm{C} 19$ & $1.0(7)$ \\
\hline $\mathrm{Cu} 1-\mathrm{N} 4-\mathrm{C} 23-\mathrm{C} 19$ & $-174.9(4)$ \\
\hline $\mathrm{C} 22-\mathrm{N} 4-\mathrm{C} 23-\mathrm{C} 24$ & $-178.1(4)$ \\
\hline $\mathrm{Cu} 1-\mathrm{N} 4-\mathrm{C} 23-\mathrm{C} 24$ & $6.0(5)$ \\
\hline $\mathrm{C} 20-\mathrm{C} 19-\mathrm{C} 23-\mathrm{N} 4$ & $-1.4(7)$ \\
\hline $\mathrm{C} 18-\mathrm{C} 19-\mathrm{C} 23-\mathrm{N} 4$ & $177.9(5)$ \\
\hline $\mathrm{C} 20-\mathrm{C} 19-\mathrm{C} 23-\mathrm{C} 24$ & $177.7(5)$ \\
\hline $\mathrm{C} 18-\mathrm{C} 19-\mathrm{C} 23-\mathrm{C} 24$ & $-3.0(7)$ \\
\hline $\mathrm{C} 13-\mathrm{N} 3-\mathrm{C} 24-\mathrm{C} 16$ & $-1.8(7)$ \\
\hline $\mathrm{Cu} 1-\mathrm{N} 3-\mathrm{C} 24-\mathrm{C} 16$ & $174.6(3)$ \\
\hline $\mathrm{C} 13-\mathrm{N} 3-\mathrm{C} 24-\mathrm{C} 23$ & $179.0(4)$ \\
\hline $\mathrm{Cu} 1-\mathrm{N} 3-\mathrm{C} 24-\mathrm{C} 23$ & $-4.6(5)$ \\
\hline $\mathrm{C} 15-\mathrm{C} 16-\mathrm{C} 24-\mathrm{N} 3$ & $2.9(7)$ \\
\hline $\mathrm{C} 17-\mathrm{C} 16-\mathrm{C} 24-\mathrm{N} 3$ & $-177.6(4)$ \\
\hline $\mathrm{C} 15-\mathrm{C} 16-\mathrm{C} 24-\mathrm{C} 23$ & $-177.9(5)$ \\
\hline $\mathrm{C} 17-\mathrm{C} 16-\mathrm{C} 24-\mathrm{C} 23$ & $1.6(7)$ \\
\hline $\mathrm{N} 4-\mathrm{C} 23-\mathrm{C} 24-\mathrm{N} 3$ & $-1.3(6)$ \\
\hline $\mathrm{C} 19-\mathrm{C} 23-\mathrm{C} 24-\mathrm{N} 3$ & $179.6(4)$ \\
\hline $\mathrm{N} 4-\mathrm{C} 23-\mathrm{C} 24-\mathrm{C} 16$ & $179.5(4)$ \\
\hline $\mathrm{C} 19-\mathrm{C} 23-\mathrm{C} 24-\mathrm{C} 16$ & $0.3(7)$ \\
\hline $\mathrm{C} 36-\mathrm{N} 5-\mathrm{C} 25-\mathrm{C} 26$ & $0.0(7)$ \\
\hline $\mathrm{Cu} 2-\mathrm{N} 5-\mathrm{C} 25-\mathrm{C} 26$ & $-179.4(4)$ \\
\hline $\mathrm{N} 5-\mathrm{C} 25-\mathrm{C} 26-\mathrm{C} 27$ & $-0.1(9)$ \\
\hline $\mathrm{C} 25-\mathrm{C} 26-\mathrm{C} 27-\mathrm{C} 28$ & $0.5(8)$ \\
\hline $\mathrm{C} 26-\mathrm{C} 27-\mathrm{C} 28-\mathrm{C} 36$ & $-0.9(8)$ \\
\hline
\end{tabular}

\begin{tabular}{|c|c|}
\hline $\mathrm{Cu} 2-\mathrm{N} 8-\mathrm{C} 47-\mathrm{C} 43$ & $-179.1(4)$ \\
\hline $\mathrm{C} 46-\mathrm{N} 8-\mathrm{C} 47-\mathrm{C} 48$ & $179.2(4)$ \\
\hline $\mathrm{Cu} 2-\mathrm{N} 8-\mathrm{C} 47-\mathrm{C} 48$ & $-1.5(5)$ \\
\hline $\mathrm{C} 44-\mathrm{C} 43-\mathrm{C} 47-\mathrm{N} 8$ & $-2.3(7)$ \\
\hline $\mathrm{C} 42-\mathrm{C} 43-\mathrm{C} 47-\mathrm{N} 8$ & $176.1(5)$ \\
\hline $\mathrm{C} 44-\mathrm{C} 43-\mathrm{C} 47-\mathrm{C} 48$ & $-179.7(5)$ \\
\hline $\mathrm{C} 42-\mathrm{C} 43-\mathrm{C} 47-\mathrm{C} 48$ & $-1.4(7)$ \\
\hline $\mathrm{C} 37-\mathrm{N} 7-\mathrm{C} 48-\mathrm{C} 40$ & $-1.5(7)$ \\
\hline $\mathrm{Cu} 2-\mathrm{N} 7-\mathrm{C} 48-\mathrm{C} 40$ & $177.9(4)$ \\
\hline $\mathrm{C} 37-\mathrm{N} 7-\mathrm{C} 48-\mathrm{C} 47$ & $179.3(4)$ \\
\hline $\mathrm{Cu} 2-\mathrm{N} 7-\mathrm{C} 48-\mathrm{C} 47$ & $-1.3(5)$ \\
\hline $\mathrm{C} 39-\mathrm{C} 40-\mathrm{C} 48-\mathrm{N} 7$ & $2.2(7)$ \\
\hline $\mathrm{C} 41-\mathrm{C} 40-\mathrm{C} 48-\mathrm{N} 7$ & $-177.1(4)$ \\
\hline $\mathrm{C} 39-\mathrm{C} 40-\mathrm{C} 48-\mathrm{C} 47$ & $-178.6(4)$ \\
\hline $\mathrm{C} 41-\mathrm{C} 40-\mathrm{C} 48-\mathrm{C} 47$ & $2.1(7)$ \\
\hline $\mathrm{N} 8-\mathrm{C} 47-\mathrm{C} 48-\mathrm{N} 7$ & $1.9(6)$ \\
\hline $\mathrm{C} 43-\mathrm{C} 47-\mathrm{C} 48-\mathrm{N} 7$ & $179.6(4)$ \\
\hline $\mathrm{N} 8-\mathrm{C} 47-\mathrm{C} 48-\mathrm{C} 40$ & $-177.3(4)$ \\
\hline $\mathrm{C} 43-\mathrm{C} 47-\mathrm{C} 48-\mathrm{C} 40$ & $0.3(7)$ \\
\hline C66-C61-C62-C63 & $-1.7(8)$ \\
\hline $\mathrm{C} 67-\mathrm{C} 61-\mathrm{C} 62-\mathrm{C} 63$ & $178.0(5)$ \\
\hline C61-C62-C63-C64 & $1.0(9)$ \\
\hline $\mathrm{C} 62-\mathrm{C} 63-\mathrm{C} 64-\mathrm{O} 63$ & $-179.5(6)$ \\
\hline $\mathrm{C} 62-\mathrm{C} 63-\mathrm{C} 64-\mathrm{C} 65$ & $0.3(9)$ \\
\hline $\mathrm{O} 63-\mathrm{C} 64-\mathrm{C} 65-\mathrm{C} 66$ & $178.9(6)$ \\
\hline $\mathrm{C} 63-\mathrm{C} 64-\mathrm{C} 65-\mathrm{C} 66$ & $-0.9(9)$ \\
\hline $\mathrm{C} 64-\mathrm{C} 65-\mathrm{C} 66-\mathrm{C} 61$ & $0.2(9)$ \\
\hline $\mathrm{C} 62-\mathrm{C} 61-\mathrm{C} 66-\mathrm{C} 65$ & $1.0(8)$ \\
\hline $\mathrm{C} 67-\mathrm{C} 61-\mathrm{C} 66-\mathrm{C} 65$ & $-178.6(5)$ \\
\hline C66-C61-C67-O61 & $9.9(8)$ \\
\hline $\mathrm{C} 62-\mathrm{C} 61-\mathrm{C} 67-\mathrm{O} 61$ & $-169.7(5)$ \\
\hline $\mathrm{C} 66-\mathrm{C} 61-\mathrm{C} 67-\mathrm{O} 62$ & $-171.1(5)$ \\
\hline $\mathrm{C} 62-\mathrm{C} 61-\mathrm{C} 67-\mathrm{O} 62$ & $9.2(8)$ \\
\hline $\mathrm{C} 76-\mathrm{C} 71-\mathrm{C} 72-\mathrm{C} 73$ & $2.7(13)$ \\
\hline $\mathrm{C} 77-\mathrm{C} 71-\mathrm{C} 72-\mathrm{C} 73$ & $178.8(8)$ \\
\hline $\mathrm{C} 71-\mathrm{C} 72-\mathrm{C} 73-\mathrm{C} 74$ & $-1.9(14)$ \\
\hline $\mathrm{C} 72-\mathrm{C} 73-\mathrm{C} 74-\mathrm{O} 73$ & $-176.2(8)$ \\
\hline $\mathrm{C} 72-\mathrm{C} 73-\mathrm{C} 74-\mathrm{C} 75$ & $-1.7(14)$ \\
\hline $\mathrm{O} 73-\mathrm{C} 74-\mathrm{C} 75-\mathrm{C} 76$ & $179.2(8)$ \\
\hline $\mathrm{C} 73-\mathrm{C} 74-\mathrm{C} 75-\mathrm{C} 76$ & $4.3(13)$ \\
\hline $\mathrm{C} 72-\mathrm{C} 71-\mathrm{C} 76-\mathrm{C} 75$ & $0.0(12)$ \\
\hline $\mathrm{C} 77-\mathrm{C} 71-\mathrm{C} 76-\mathrm{C} 75$ & $-176.2(7)$ \\
\hline $\mathrm{C} 74-\mathrm{C} 75-\mathrm{C} 76-\mathrm{C} 71$ & $-3.5(12)$ \\
\hline $\mathrm{C} 72-\mathrm{C} 71-\mathrm{C} 77-\mathrm{O} 71$ & $174.9(8)$ \\
\hline $\mathrm{C} 76-\mathrm{C} 71-\mathrm{C} 77-\mathrm{O} 71$ & $-8.9(11)$ \\
\hline $\mathrm{C} 72-\mathrm{C} 71-\mathrm{C} 77-\mathrm{O} 72$ & $-7.1(12)$ \\
\hline $\mathrm{C} 76-\mathrm{C} 71-\mathrm{C} 77-\mathrm{O} 72$ & $169.1(8)$ \\
\hline
\end{tabular}


Hydrogen-bond geometry $\left(\AA,{ }^{\circ}\right)$

\begin{tabular}{lllll}
\hline$D-\mathrm{H} \cdots A$ & $D-\mathrm{H}$ & $\mathrm{H} \cdots A$ & $D \cdots A$ & $D-\mathrm{H}^{\cdots} A$ \\
\hline $\mathrm{O} 1 W-\mathrm{H} 1 A \cdots \mathrm{O} 53$ & 0.97 & 1.90 & $2.819(5)$ & 157 \\
$\mathrm{O} 1 W-\mathrm{H} 1 B \cdots \mathrm{O} 61^{\mathrm{i}}$ & 1.00 & 1.76 & $2.758(5)$ & 169 \\
$\mathrm{O} 2 W-\mathrm{H} 2 A \cdots \mathrm{O} 61^{\mathrm{i}}$ & 0.97 & 1.75 & $2.708(5)$ & 169 \\
$\mathrm{O} 2 W-\mathrm{H} 2 B \cdots \mathrm{O} 4 W^{\mathrm{ii}}$ & 0.83 & 2.04 & $2.843(6)$ & 160 \\
$\mathrm{O} 3 W-\mathrm{H} 3 A \cdots \mathrm{O} 62^{\mathrm{iii}}$ & 1.00 & 1.69 & $2.685(5)$ & 172 \\
$\mathrm{O} 3 W-\mathrm{H} 3 B \cdots \mathrm{O} 62$ & 1.00 & 1.84 & $2.731(7)$ & 147 \\
$\mathrm{O} 4 W-\mathrm{H} 4 A \cdots \mathrm{O} 1 W$ & 1.01 & 1.79 & $2.756(6)$ & 160 \\
$\mathrm{O} 4 W-\mathrm{H} 4 B \cdots \mathrm{O} 3 W$ & 0.86 & 1.94 & $2.750(7)$ & 157 \\
$\mathrm{O} 5 W-\mathrm{H} 5 A \cdots \mathrm{O} 72$ & 0.96 & 1.87 & $2.818(8)$ & 169 \\
$\mathrm{O} 5 W-\mathrm{H} 5 B \cdots \mathrm{O} 6 W^{\text {iv }}$ & 0.97 & 1.84 & $2.779(7)$ & 164 \\
$\mathrm{O} 73-\mathrm{H} 73 \cdots \mathrm{O} 8 W$ & 0.82 & 2.15 & $2.88(2)$ & 148 \\
$\mathrm{O} 63-\mathrm{H} 63 \cdots \mathrm{O} 2 W$ & 0.82 & 1.85 & $2.638(5)$ & 161 \\
$\mathrm{C} 3-\mathrm{H} 3 \cdots \mathrm{O} 1 W^{\mathrm{i}}$ & 0.93 & 2.59 & $3.240(6)$ & 127 \\
$\mathrm{C} 8-\mathrm{H} 8 \cdots \mathrm{O} 6 W$ & 0.93 & 2.48 & $3.371(8)$ & 162 \\
$\mathrm{C} 14-\mathrm{H} 14 \cdots \mathrm{O} 72$ & 0.93 & 2.50 & $3.388(10)$ & 159 \\
$\mathrm{C} 21-\mathrm{H} 21 \cdots \mathrm{O} 4 W^{\mathrm{ii}}$ & 0.93 & 2.58 & $3.230(8)$ & 128 \\
$\mathrm{C} 25-\mathrm{H} 25 \cdots \mathrm{O} 52$ & 0.93 & 2.47 & $3.033(6)$ & 119 \\
$\mathrm{C} 33-\mathrm{H} 33 \cdots \mathrm{O} 62^{\mathrm{v}}$ & 0.93 & 2.55 & $3.247(8)$ & 132 \\
$\mathrm{C} 38-\mathrm{H} 38 \cdots \mathrm{O} 61^{\mathrm{iii}}$ & 0.93 & 2.37 & $3.298(8)$ & 172 \\
$\mathrm{C} 65-\mathrm{H} 65 \cdots \mathrm{O} 63^{\text {vi }}$ & 0.93 & 2.49 & $3.410(6)$ & 172 \\
$\mathrm{C} 73-\mathrm{H} 71 \cdots \mathrm{O} 7 W^{\text {iv }}$ & 0.93 & 2.58 & $3.413(13)$ & 149 \\
& & & & \\
\hline
\end{tabular}

Symmetry codes: (i) $x,-y+1 / 2, z+1 / 2$; (ii) $-x+1, y+1 / 2,-z+1 / 2$; (iii) $-x+1,-y,-z$; (iv) $x,-y+1 / 2, z-1 / 2$; (v) $-x+1, y-1 / 2,-z+1 / 2$; (vi) $-x+1,-y+1,-z$. 